CUBO A Mathematical Journal Vol.13, № 02, (85-117). June 2011

\title{
Differential forms versus multi-vector functions in Hermitean Clifford analysis
}

\author{
F. Brackx, H. De Schepper and V. Souček \\ Ghent University \\ Faculty of Engineering \\ Department of Mathematical Analysis \\ Gent, Belgium \\ email: fb@cage.ugent.be \\ and \\ F. Brackx, H. De Schepper and V. Souček \\ Charles University \\ Faculty of Mathematics and Physics \\ Praha, Czech Republic
}

\begin{abstract}
Similarities are shown between the algebras of complex differential forms and of complex Clifford algebra-valued multi-vector functions in an open region of Euclidean space of even dimension.
\end{abstract}

\section{RESUMEN}

Se presentan las similitudes entre las álgebras de formas diferenciales complejas y de las funciones de álgebras de Clifford complejas con valores de múltiples vectores aplicados en una región abierta del espacio euclidiano de dimensión par. 
Keywords and phrases: complex differential forms, multi-vector functions, Hermitean Clifford analysis.

Mathematics Subject Classification: 30G35

\section{Introduction}

Usually Clifford analysis is understood to be the study of the solutions of the Dirac equation for functions defined on the (anti-)Euclidean vector space $\mathbb{R}^{0, m}$ and taking values in the corresponding Clifford algebra $\mathbb{R}_{0, m}$. It thus offers a proper analogue to the Cauchy-Riemann equations for holomorphic functions in the complex plane. For a thorough study of the so-called monogenic functions of Clifford analysis we refer to the standard textbooks [5, 15, 17, 18.

The symmetry group of the Dirac equation is either $\mathrm{SO}(\mathrm{m})$ or $\operatorname{Spin}(\mathrm{m})$, according to the definition of the group action on the values taken by the functions under consideration. If these values are in the Clifford algebra with left multiplication, the symmetry group is $\operatorname{Spin}(\mathrm{m})$, which then usually is realized inside the Clifford algebra. In the case of functions with values in the Clifford algebra with both side action, it is more natural to identify the Dirac equation with the equation $\left(d+d^{*}\right) f=0$, and to identify the space of values, in casu the Clifford algebra, as a vector space, with the Grassmann algebra of $\mathbb{R}^{\mathrm{m}}$. This Grassmann algebra may then be decomposed into the direct sum of its homogeneous parts, which is a decomposition into irreducible parts under the action of $\mathrm{SO}(\mathrm{m})$. In this framework it was shown (see [13]) that, on the polynomial level, the space of monogenic functions can be split into a direct sum of solutions of the Hodge-de Rham equations for homogeneous differential forms. This entails a finer structure of the space of monogenic functions, which manifests itself explicitly in a finer form of the corresponding Fischer decomposition (see [14]).

An important ingredient in the latter approach is the translation of spaces and operators from the language of multivector functions with values in a Clifford algebra to the language of real differential forms, as was described in detail in [6]. Let us give a very brief overview. On the one hand we have the Cartan algebra $\bigwedge(\mathrm{G})$ of smooth real differential forms in an open subset $\mathrm{G}$ of Euclidean space $\mathbb{R}^{m}$, endowed with exterior multiplication. A fundamental operator on $\bigwedge(G)$ is the exterior derivative $d$ with its important property that for any differential form $\omega, d^{2} \omega=d(d \omega)=0$. Introducing the Hodge co-derivative $\mathrm{d}^{*}$ leads to the differential operator $\mathrm{D}=\mathrm{d}+\mathrm{d}^{*}$, by means of which the so-called "harmonic" $r$-forms $(0<r<m)$ are characterized as smooth differential $r$ forms $\omega^{r}$ satisfying $D \omega^{r}=0$. On the other hand we have the algebra $\mathcal{E}(G)$ of smooth multi-vector functions in $\mathrm{G}$. Multi-vector functions arise in a natural way when considering functions defined in $G$ and taking values in the universal real Clifford algebra $\mathbb{R}_{0, m}$ constructed over $\mathbb{R}^{0, m}$, i.e. $\mathbb{R}^{m}$ equipped with an anti-Euclidean metric. If $\mathbb{R}_{0, m}^{r}(0 \leq r \leq m)$ denotes the space of $r$-vectors, then the Clifford algebra $\mathbb{R}_{0, m}$ is precisely the associative algebra $\mathbb{R}_{0, m}=\bigoplus_{r=0}^{m} \mathbb{R}_{0, m}^{r}$, and an r-vector function $F_{r}$ is a map $F_{r}: G \rightarrow \mathbb{R}_{0, m}^{r}$. A fundamental operator on the space of smooth multi-vector 
functions, is the rotation-invariant Dirac operator $\partial_{\underline{x}}$, by means of which the so-called monogenic functions are characterized as the smooth functions $f$ satisfying $\partial_{\underline{x}} f=0$, as already mentioned above. The spaces of smooth differential forms and of smooth multi-vector functions were shown to be isomorphic in a natural way: a smooth $r$-form is identified with a smooth $r$-vector function, and the action of the differential operator $d+d^{*}$ on the space $\Lambda^{r}(G)$ of smooth $r$-forms, is identified with the action of the Dirac operator $\partial_{\underline{x}}$ on the space $\mathcal{E}_{\mathrm{r}}(G)$ of smooth $r$-vector functions. Also other correspondences were studied in detail in [6].

When the dimension is taken to be even $(m=2 n)$, one can make the framework of Clifford analysis closer to complex analysis by introducing on $\mathbb{R}^{2 n}$ a complex structure J. The symmetry group then reduces to the subgroup $\mathrm{U}(n) \subset \mathrm{SO}(2 \mathrm{n})$ preserving the chosen complex structure $\mathrm{J}$. This is the basic setting for so-called Hermitean Clifford analysis, which recently has emerged as a new and successful branch of Clifford analysis, offering yet a refinement of the Euclidean case. The functions studied are defined in open regions of $\mathbb{C}^{n}$ and take their values in the complex Clifford algebra $\mathbb{C}_{2 n}$. More particularly Hermitean Clifford analysis focusses on the simultaneous null solutions, called Hermitean (or $\mathrm{h}-$ ) monogenic functions, of two Hermitean Dirac operators $\partial_{\underline{z}}$ and $\partial_{z^{\dagger}}$. A systematic development of this function theory, including the invariance properties with respect to the underlying Lie groups and Lie algebras, is still in full progress, see e.g. [9, 1, 2, 7, 8, 3, 4, 23, 12. Part of this program also concerns the study of the finer structure induced on the space of monogenic functions by the choice of the complex structure J.

When studying the Dirac equation for functions with values in a Clifford algebra, it is well known that the Clifford algebra can be split into the direct sum of a number of isomorphic copies of the basic spinor representation. Accordingly, the set of equations will split into a number of independent subsets of equations for functions with values in the various copies of spinor space. It is a trivial observation that all these subsystems are equivalent to each other and their solutions will have the same properties, whence, without any loss of generality, we can restrict the study to functions with values in the space of spinors (or half-spinors in even dimension). In the standard situation, this space of values cannot be split further since they are already irreducible under the (left) action of the $\operatorname{Spin}(\mathrm{m})$ group. However, after having fixed the complex structure J, the symmetry group is reduced, as explained above, and the spinor space decomposes further into smaller pieces. If it is realized in a standard way as the Grassmann algebra over the maximal isotropic subspace in $\mathbb{C}^{2 n}$, then this splitting is just the splitting into homogeneous components; for details see e.g. [2].

Our final aim is to understand the finer structure of the space of monogenic functions induced by this splitting. A first step towards that goal is to establish a scheme for the translation of spaces and operators between the language of complex Clifford algebra and the language of complex differential forms. In fact this is the complex analogue of the translation in the Euclidean situation 
mentioned above, see [6]. The purpose of the underlying paper is precisely to describe in a rather formal, yet detailed, way the similarities between complex differential forms in open regions of $\mathbb{C}^{n}$ on the one hand and multivector functions in the Hermitean Clifford analysis setting on the other. Crucial to this description is the detailed analysis of the structure of complex Clifford algebra as carried out in [10]. The Hermitean Dirac operators $\partial_{\underline{z}}$ and $\partial_{\underline{z}} \dagger$ and the associated operators $\partial_{\underline{z}} \bullet$, $\partial_{\underline{z}} \wedge, \partial_{\underline{z}^{\dagger}} \bullet$ and $\partial_{\underline{z}^{\dagger}} \wedge$, originating by splitting the Clifford or geometric product into its "inner" or "dot" and "outer" or "wedge" parts, are identified with well-known differential operators for complex differential forms on Kählerian manifolds in $\mathbb{C}^{n}$. However it should be emphasized that, in this paper, we restrict ourselves to the flat Kählerian metric on $\mathbb{C}^{\mathrm{n}}$ with fundamental form $\Omega=\frac{i}{2} \partial \bar{\partial}|\underline{z}|^{2}$. The more general approach of Hermitean Clifford analysis on complex Hermitean manifolds and its comparison with complex analysis on Kählerian manifolds is the subject of the forthcoming paper [1].

The paper is structured as follows. Sections 2 and 3 are introductory, fixing our definitions and notations. An identification of all differential operators and forms under consideration in both pictures is described in Section 4. The relation to the operators which are standard in Kählerian geometry is clarified in Section 5. The last section adds some remarks on the Hodge operator.

\section{Multi-vector functions: preliminaries}

In this section we recall some basic notions and results from Clifford algebra and Clifford analysis.

The construction of the universal real Clifford algebra is well-known; for an in-depth study we refer the reader to e.g. [22]. Here we restrict ourselves to a schematic approach. Let $\mathbb{R}^{0, m}$ be the real vector space $\mathbb{R}^{m}(m \geq 1)$ endowed with a non-degenerate symmetric bilinear form $\mathcal{B}$ of signature $(0, m)$, and let $\left(e_{1}, \ldots, e_{m}\right)$ be an associated orthonormal basis, i.e.

$$
\mathcal{B}\left(e_{i}, e_{j}\right)=\left\{\begin{aligned}
-1 & \text { if } \quad i=j \\
0 & \text { if } \quad i \neq j
\end{aligned} \quad(1 \leq i, j \leq m)\right.
$$

then the anti-Euclidean metric on $\mathbb{R}^{0, m}$ is induced by the scalar product

$$
\left\langle e_{i}, e_{j}\right\rangle=-\mathcal{B}\left(e_{i}, e_{j}\right)=\delta_{i j}, \quad 1 \leq i, j \leq m
$$

We first introduce the anti-symmetric outer product by the rules

$$
\begin{aligned}
e_{i} \wedge e_{i} & =0, \quad 1 \leq i \leq m \\
e_{i} \wedge e_{j}+e_{j} \wedge e_{i} & =0, \quad 1 \leq i \neq j \leq m
\end{aligned}
$$

and for each $A=\left\{i_{1}, i_{2}, \ldots, i_{r}\right\} \subset M=\{1, \ldots, m\}$, with $1 \leq i_{1}<i_{2}<\ldots<i_{r} \leq m$, i.e. ordered in the natural way, we put

$$
e_{\mathrm{A}}=e_{i_{1}} \wedge e_{i_{2}} \wedge \ldots \wedge e_{i_{r}}
$$


while $e_{\emptyset}=1$. Then for each $r=0,1, \ldots, m$, the set $\left\{e_{A}: A \subset M\right.$ and $\left.|A|=r\right\}$ is a basis for the space $\mathbb{R}_{0, m}^{r}$ of so-called $r$-vectors. Next, we introduce the inner product

$$
e_{i} \cdot e_{j}=-\left\langle e_{i}, e_{j}\right\rangle=\mathcal{B}\left(e_{i}, e_{j}\right)=-\delta_{i j}, \quad 1 \leq i, j \leq m
$$

leading to the so-called geometric product of vectors in the Clifford algebra:

$$
e_{i} e_{j}=e_{i} \cdot e_{j}+e_{i} \wedge e_{j}, \quad 1 \leq i, j \leq m
$$

The respective definitions of the inner, the outer and the geometric product are then extended to $r$-vectors as follows: for the inner product, we have

$$
e_{j} \cdot e_{A}=e_{j} \cdot\left(e_{i_{1}} \wedge \ldots \wedge e_{i_{r}}\right)=\sum_{k=1}^{r}(-1)^{k} \delta_{j i_{k}} e_{A \backslash\left\{i_{k}\right\}}
$$

with

$$
e_{A \backslash\left\{i_{k}\right\}}=e_{i_{1}} \wedge \ldots \wedge e_{i_{k-1}} \wedge\left[e_{i_{k}} \wedge\right] e_{i_{k+1}} \wedge \ldots \wedge e_{i_{r}}
$$

while for the outer product

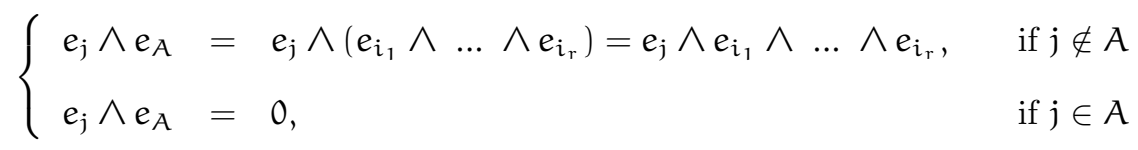

and finally, for the geometric product (or product for short)

$$
e_{j} e_{A}=e_{j} \cdot e_{A}+e_{j} \wedge e_{A}
$$

Finally, these definitions are linearly extended to the whole of the Clifford algebra $\mathbb{R}_{0, m}$, which is the associative algebra

$$
\mathbb{R}_{0, m}=\bigoplus_{r=0}^{m} \mathbb{R}_{0, m}^{r}
$$

If $[\cdot]_{r}: \mathbb{R}_{0, m} \rightarrow \mathbb{R}_{0, m}^{r}$ denotes the projection operator from $\mathbb{R}_{0, m}$ onto $\mathbb{R}_{0, m}^{r}$, then each Clifford number $a \in \mathbb{R}_{0, m}$ may be written as

$$
\mathrm{a}=\sum_{\mathrm{r}=0}^{\mathrm{m}}[\mathrm{a}]_{\mathrm{r}}
$$

Note that in particular for a 1 -vector $u$ and an r-vector $v_{r}$, one has

$$
u v_{r}=u \cdot v_{r}+u \wedge v_{r}
$$

with

$$
\begin{aligned}
& u \cdot v_{r}=\left[u v_{r}\right]_{r-1}=\frac{1}{2}\left(u v_{r}-(-1)^{r} v_{r} u\right) \\
& u \wedge v_{r}=\left[u v_{r}\right]_{r+1}=\frac{1}{2}\left(u v_{r}+(-1)^{r} v_{r} u\right)
\end{aligned}
$$


Usually $\mathbb{R}$ and $\mathbb{R}^{m}$ are identified with $\mathbb{R}_{0, m}^{0}$ and $\mathbb{R}_{0, m}^{1}$ respectively. An element $X=\left(X_{1}, \ldots, X_{m}\right) \in$ $\mathbb{R}^{m}$ is thus identified with the 1 -vector $\underline{X}=\sum_{j=1}^{m} X_{j} e_{j}$.

Now let $G$ be an open region in $\mathbb{R}^{m}$. A smooth $r$-vector function $F_{r}$ is a map

$$
\mathrm{F}_{\mathrm{r}}: \mathrm{G} \rightarrow \mathbb{R}_{0, \mathrm{~m}}^{\mathrm{r}}, \underline{X} \mapsto \sum_{|\mathrm{A}|=\mathrm{r}} \mathrm{F}_{\mathrm{r}, \mathrm{A}}(\underline{\mathrm{X}}) \mathrm{e}_{\mathrm{A}}
$$

where for each $A, F_{r, A}$ is a smooth real valued function in $G$. We denote by $\mathcal{E}_{r}(G)$ the space of smooth r-vector functions in $\mathrm{G}$, and we put

$$
\mathcal{E}(\mathrm{G})=\bigoplus_{\mathrm{r}=0}^{\mathrm{m}} \mathcal{E}_{\mathrm{r}}(\mathrm{G})
$$

The projection operator from $\mathcal{E}(\mathrm{G})$ onto $\mathcal{E}_{\mathrm{r}}(\mathrm{G})$ is denoted by $[.]_{\mathrm{r}}$.

A fundamental operator in Clifford analysis is the so-called Dirac operator, a first order vector valued differential operator given by

$$
\partial_{\underline{x}}=\sum_{j=1}^{m} e_{j} \partial_{x_{j}}
$$

Since the multiplication in the Clifford algebra is non-commutative, operators can act from the left or from the right on a function. For the Dirac operator and a function $F=\sum_{A} e_{A} F_{A} \in \mathcal{E}(G)$, these actions are given by

$$
\partial_{\underline{X}} F=\sum_{j=1}^{m} \sum_{A} e_{j} e_{A} \partial X_{X_{j}} F_{A} \quad \text { and } \quad F \partial_{\underline{X}}=\sum_{j=1}^{m} \sum_{A} e_{A} e_{j} \partial_{X_{j}} F_{A}
$$

A function $F \in \mathcal{E}(\mathrm{G})$ is called left (resp. right) monogenic in $\mathrm{G}$ if and only if it satisfies in $\mathrm{G}$ the equation $\partial_{\underline{x}} F=0\left(\right.$ resp. $\left.F \partial_{\underline{x}}=0\right)$.

Restricting the Dirac operator $\partial_{\underline{x}}$ to the space $\mathcal{E}_{\mathrm{r}}(\mathrm{G})$, we find for an r-vector function $\mathrm{F}_{\mathrm{r}}$ that $\partial_{\underline{X}} F_{r}$ and $F_{r} \partial_{\underline{X}}$ split into an $(r-1)$-vector part and an $(r+1)$-vector part:

$$
\begin{aligned}
& \partial_{\underline{X}} F_{r}=\sum_{j=1}^{m} e_{j} \partial_{X_{j}} F_{r}=\sum_{j=1}^{m} e_{j} \bullet \partial_{X_{j}} F_{r}+\sum_{j=1}^{m} e_{j} \wedge \partial_{X_{j}} F_{r} \\
& F_{r} \partial_{\underline{X}}=\sum_{j=1}^{m} \partial_{X_{j}} F_{r} e_{j}=\sum_{j=1}^{m} \partial_{X_{j}} F_{r} \bullet e_{j}+\sum_{j=1}^{m} \partial_{X_{j}} F_{r} \wedge e_{j}
\end{aligned}
$$


It readily follows that

$$
\begin{aligned}
& {\left[\underline{\partial}_{\underline{X}} F_{r}\right]_{r-1}=\sum_{j=1}^{m} e_{j} \cdot \partial x_{j} F_{r}=(-1)^{r+1} \sum_{j=1}^{m} \partial_{X_{j}} F_{r} \bullet e_{j}=(-1)^{r+1}\left[F_{r} \partial \underline{x}\right]_{r-1}} \\
& {\left[\partial_{\underline{X}} F_{r}\right]_{r+1}=\sum_{j=1}^{m} e_{j} \wedge \partial_{X_{j}} F_{r}=(-1)^{r} \quad \sum_{j=1}^{m} \partial_{X_{j}} F_{r} \wedge e_{j}=(-1)^{r} \quad\left[F_{r} \partial \underline{X}\right]_{r+1}}
\end{aligned}
$$

Usually one introduces the notations

$$
\begin{array}{ll}
\partial_{\underline{x}} \bullet F_{r}=\left[\partial_{\underline{x}} F_{r}\right]_{r-1}, & \partial \underline{x} \wedge F_{r}=\left[\partial_{\underline{x}} F_{r}\right]_{r+1} \\
F_{r} \cdot \partial \underline{x}=\left[F_{r} \partial \underline{x}\right]_{r-1}, & F_{r} \wedge \partial_{\underline{x}}=\left[F_{r} \partial \underline{x}\right]_{r+1}
\end{array}
$$

The action of the Dirac operator $\partial_{\underline{x}}$ on $\mathcal{E}_{\mathrm{r}}(\mathrm{G})$ thus gives rise to two auxiliary differential operators

$$
\begin{aligned}
\partial_{\underline{x}} \bullet: \mathcal{E}_{r}(G) \rightarrow \mathcal{E}_{r-1}(G) ; F_{r} & \mapsto\left(\partial_{\underline{x}} \bullet\right) F_{r}=\partial \underline{x} \bullet F_{r}=\left[\partial_{\underline{x}} F_{r}\right]_{r-1} \\
\partial_{\underline{x}} \wedge: \mathcal{E}_{r}(G) \rightarrow \mathcal{E}_{r+1}(G) ; F_{r} & \mapsto\left(\partial_{\underline{x}} \wedge\right) F_{r}=\partial_{\underline{x}} \wedge F_{r}=\left[\partial_{\underline{x}} F_{r}\right]_{r+1}
\end{aligned}
$$

for which it holds that

$$
\partial \underline{x}=\partial \underline{x} \bullet+\partial_{\underline{x}} \wedge
$$

Symbolically these operators may be written as

$$
\begin{aligned}
& \left(\partial_{\underline{x}} \bullet\right)=\sum_{j=1}^{m}\left(e_{j} \bullet\right) \partial_{x_{j}} \\
& \left(\partial_{\underline{x}} \wedge\right)=\sum_{j=1}^{m}\left(e_{j} \wedge\right) \partial_{x_{j}}
\end{aligned}
$$

Their action on $\mathcal{E}_{\mathrm{r}}(\mathrm{G})$ is two-fold in the sense that they act on the multi-vector by means of the inner and outer product with basis vectors, and at the same time on the function coefficients by partial differentiation. We thus have that, for a smooth $r$-vector function $F_{r}$, the notions of left monogenicity and right monogenicity coincide, and moreover that $F_{r}$ is left as well as right monogenic in $G$ if and only if in $G$

$$
\partial_{\underline{x}} F_{r}=0 \Longleftrightarrow F_{r} \partial_{\underline{x}}=0 \Longleftrightarrow\left\{\begin{array}{l}
\partial_{\underline{x}} \bullet F_{r}=0 \\
\partial_{\underline{x}} \wedge F_{r}=0
\end{array}\right.
$$

As the Dirac operator $\partial_{\underline{x}}$ factorizes the Laplace operator, viz

$$
\partial_{\underline{\underline{x}}}^{2}=\partial_{\underline{x}} \cdot \partial_{\underline{x}}+\partial_{\underline{x}} \wedge \partial_{\underline{x}}=\partial_{\underline{x}} \cdot \partial \underline{x}=-\left\langle\partial_{\underline{x}}, \partial \underline{x}\right\rangle=-\Delta_{m}
$$

a monogenic function in $\mathrm{G}$ is also harmonic in $\mathrm{G}$, but the converse clearly is not true. As moreover

$$
\left(\partial_{\underline{x}} \bullet\right)^{2}=\left(\partial_{\underline{x}} \wedge\right)^{2}=0
$$


we have that

$$
-\Delta_{m}=\left(\partial_{\underline{x}} \bullet+\partial_{\underline{x}} \wedge\right)^{2}=\partial_{\underline{x}} \bullet \partial_{\underline{x}} \wedge+\partial_{\underline{x}} \wedge \partial_{\underline{x}} \bullet
$$

the two second order differential operators $\left(\partial_{\underline{x}} \bullet \partial_{\underline{X}} \wedge\right)$ and $\left(\partial_{\underline{x}} \wedge \partial_{\underline{x}} \bullet\right)$ arising above being scalar operators in the sense that they keep the order of the multi-vector function invariant. However the function coefficients, while being differentiated, are interchanged w.r.t. the basis multi-vectors.

When allowing for complex constants and moreover taking the dimension to be even: $\mathrm{m}=2 \mathrm{n}$, the same generators $\left(e_{1}, \ldots, e_{n}, e_{n+1}, \ldots, e_{2 n}\right)$ produce the complex Clifford algebra $\mathbb{C}_{2 n}$, which is the complexification of the real Clifford algebra $\mathbb{R}_{0,2 n}$, i.e. $\mathbb{C}_{2 n}=\mathbb{R}_{0,2 n} \oplus i \mathbb{R}_{0,2 n}$. Any complex Clifford number $\lambda \in \mathbb{C}_{2 n}$ may thus be written as $\lambda=a+i b, a, b \in \mathbb{R}_{0,2 n}$, an observation leading to the definition of the Hermitean conjugation $\lambda^{\dagger}=(a+i b)^{\dagger}=\bar{a}-i \bar{b}$, where the bar notation stands for the usual Clifford conjugation in $\mathbb{R}_{0,2 n}$, i.e. the main anti-involution for which $\bar{e}_{j}=-e_{j}, j=1, \ldots, 2 n$. This Hermitean conjugation also leads to a Hermitean inner product and its associated norm on $\mathbb{C}_{2 n}$ given by $(\lambda, \mu)=\left[\lambda^{\dagger} \mu\right]_{0}$ and $|\lambda|=\sqrt{\left[\lambda^{\dagger} \lambda\right]_{0}}=\left(\sum_{A}\left|\lambda_{A}\right|^{2}\right)^{1 / 2}$.

This is the framework for so-called Hermitean Clifford analysis, a refinement of Euclidean Clifford analysis. An elegant way of introducing this setting consists in considering a so-called complex structure, i.e. a specific $\mathrm{SO}\left(2 \mathrm{n} ; \mathbb{R}\right.$ )-element J for which it holds that $\mathrm{J}^{2}=-\mathbf{1}$ ( $\mathrm{see}[1,2$, $)$. Here, $J$ is chosen to act upon the generators $e_{1}, \ldots, e_{2 n}$ of the Clifford algebra as

$$
J\left[e_{j}\right]=-e_{n+j} \quad \text { and } \quad J\left[e_{n+j}\right]=e_{j}, \quad j=1, \ldots, n
$$

With J one may associate two projection operators $\frac{1}{2}(\mathbf{1} \pm i J)$ which produce the main objects of the Hermitean setting by acting upon the corresponding objects in the Euclidean framework. First of all, the so-called Witt basis elements $\left(\mathfrak{f}_{j}, \mathfrak{f}_{j}^{\dagger}\right)_{j=1}^{n}$ for $\mathbb{C}_{2 n}$ are obtained through the action of $\pm \frac{1}{2}(\mathbf{1} \pm \mathrm{iJ})$ on the original orthogonal basis:

$$
\begin{aligned}
& \mathfrak{f}_{j}=\frac{1}{2}(1+i J)\left[e_{j}\right]=\frac{1}{2}\left(e_{j}-i e_{n+j}\right), \quad j=1, \ldots, n \\
& \mathfrak{f}_{j}^{\dagger}=-\frac{1}{2}(1-i J)\left[e_{j}\right]=-\frac{1}{2}\left(e_{j}+i e_{n+j}\right), \quad j=1, \ldots, n
\end{aligned}
$$

The Witt basis elements satisfy the Grassmann identities

$$
\mathfrak{f}_{j} \mathfrak{f}_{k}+\mathfrak{f}_{k} \mathfrak{f}_{j}=\mathfrak{f}_{j}^{\dagger} \mathfrak{f}_{k}^{\dagger}+\mathfrak{f}_{k}^{\dagger} \mathfrak{f}_{j}^{\dagger}=0, \quad j, k=1, \ldots, n
$$

including their isotropy: $\mathfrak{f}_{j}^{2}=\mathfrak{f}_{j}^{\dagger^{2}}=0, \mathfrak{j}=1, \ldots, n$, as well as the duality identities

$$
\mathfrak{f}_{j} \mathfrak{f}_{k}^{\dagger}+\mathfrak{f}_{k}^{\dagger} \mathfrak{f}_{j}=\delta_{j k}, j, k=1, \ldots, n
$$

The Witt basis of the complex Clifford algebra $\mathbb{C}_{2 n}$ is then obtained, in much the same way as is done for the basis of the real Clifford algebra, by taking all possible products of Witt basis vectors. 
Introducing the inner and outer products for the Witt basis vectors we have, see also [9],

$$
\begin{array}{rlrl}
\mathfrak{f}_{j} \bullet \mathfrak{f}_{\mathrm{k}} & =\mathfrak{f}_{j}^{\dagger} \bullet \mathfrak{f}_{\mathrm{k}}^{\dagger}=0, & \mathrm{j}, \mathrm{k}=1, \ldots, \mathrm{n} \\
\mathfrak{f}_{j} \bullet \mathfrak{f}_{\mathrm{k}}^{\dagger}=\mathfrak{f}_{j}^{\dagger} \bullet \mathfrak{f}_{\mathrm{k}}=\frac{1}{2} \delta_{j k}, & j, k=1, \ldots, n
\end{array}
$$

and

$$
\begin{aligned}
\mathfrak{f}_{j} \wedge \mathfrak{f}_{k}=-\mathfrak{f}_{k} \wedge \mathfrak{f}_{j}, & \mathrm{j}, \mathrm{k}=1, \ldots, \mathrm{n} \\
\mathfrak{f}_{j}^{\dagger} \wedge \mathfrak{f}_{k}^{\dagger}=-\mathfrak{f}_{k}^{\dagger} \wedge \mathfrak{f}_{j}^{\dagger}, & j, k=1, \ldots, n
\end{aligned}
$$

eventually yielding

$$
\begin{array}{lll}
\mathfrak{f}_{\mathfrak{j}} \mathfrak{f}_{k}=\mathfrak{f}_{j} \bullet \mathfrak{f}_{k}+\mathfrak{f}_{j} \wedge \mathfrak{f}_{k}=\mathfrak{f}_{j} \wedge \mathfrak{f}_{k}, & j, k=1, \ldots, n \\
\mathfrak{f}_{j}^{\dagger} \mathfrak{f}_{k}^{\dagger}=\mathfrak{f}_{j}^{\dagger} \bullet \mathfrak{f}_{k}^{\dagger}+\mathfrak{f}_{j}^{\dagger} \wedge \mathfrak{f}_{k}^{\dagger}=\mathfrak{f}_{j}^{\dagger} \wedge \mathfrak{f}_{k}^{\dagger}, & j, k=1, \ldots, n \\
\mathfrak{f}_{j} \mathfrak{f}_{k}^{\dagger}=\mathfrak{f}_{j} \bullet \mathfrak{f}_{k}^{\dagger}+\mathfrak{f}_{j} \wedge \mathfrak{f}_{k}^{\dagger}=\frac{1}{2} \delta_{j k}+\mathfrak{f}_{j} \wedge \mathfrak{f}_{k}^{\dagger}, & j, k=1, \ldots, n
\end{array}
$$

This leads to the Grassmann structure of the complex Clifford algebra

$$
\mathbb{C}_{2 n} \cong \bigoplus_{p=0}^{n} \bigoplus_{q=0}^{n} \bigwedge_{2 n}^{p, q}
$$

where

$$
\bigwedge_{2 \mathrm{n}}^{\mathrm{p}, \mathrm{q}}=\operatorname{span}_{\mathbb{C}}\left\{\mathfrak{f}_{j_{1}}^{\dagger} \wedge \ldots \wedge \mathfrak{f}_{j_{p}}^{\dagger} \wedge \mathfrak{f}_{k_{1}} \wedge \ldots \wedge \mathfrak{f}_{k_{q}} \mid j_{1}<j_{2}<\ldots<j_{p}, k_{1}<k_{2}<\ldots<k_{q}\right\}
$$

A vector $\left(X_{1}, \ldots, X_{2 n}\right)$ in $\mathbb{R}^{0,2 n}$ is now denoted by $\left(x_{1}, \ldots, x_{n}, y_{1}, \ldots, y_{n}\right)$ and is identified with the Clifford vector $\underline{X}=\sum_{j=1}^{n}\left(e_{j} x_{j}+e_{n+j} y_{j}\right)$; the action of the complex structure $J$ on $\underline{X}$ yields the twisted vector

$$
\underline{X} \mid=J[\underline{X}]=\sum_{j=1}^{n}\left(e_{j} y_{j}-e_{n+j} x_{j}\right)
$$

Note that $\underline{X}$ and $\underline{X} \mid$ anti-commute, since they are orthogonal w.r.t. the standard Euclidean scalar product; more precisely they satisfy the following properties.

Lemma 2.1. One has

(i) $\underline{X} \bullet \underline{X} \mid=0$

(ii) $\underline{x} \wedge \underline{X} \mid=\sum_{j \neq k} x_{j} y_{k}\left(e_{j} e_{k}-e_{n+k} e_{n+j}\right)-\sum_{j, k} e_{j} e_{n+k}\left(x_{j} x_{k}+y_{j} y_{k}\right)$

(iii) $\underline{X} \mid \wedge \underline{X}=\sum_{j \neq k} x_{j} y_{k}\left(e_{k} e_{j}-e_{n+j} e_{n+k}\right)-\sum_{j, k} e_{n+k} e_{j}\left(x_{j} x_{k}+y_{j} y_{k}\right)$

(iv) $\underline{X} \underline{X}|+\underline{X}| \underline{X}=\underline{X} \wedge \underline{X}|+\underline{X}| \wedge \underline{X}=0$ 
The actions of the projection operators on the Clifford vector $\underline{X}$ then produce the mutually Hermitean conjugate Hermitean Clifford variables $\underline{z}$ and $\underline{z}^{\dagger}$, i.e.

$$
\begin{aligned}
\underline{z} & =\frac{1}{2}(1+i J)[\underline{X}]=\frac{1}{2}(\underline{X}+i \underline{X} \mid) \\
\underline{z}^{\dagger} & =-\frac{1}{2}(1-i J)[\underline{X}]=-\frac{1}{2}(\underline{X}-i \underline{X} \mid)
\end{aligned}
$$

which may also be rewritten in terms of the Witt basis elements as

$$
\underline{z}=\sum_{j=1}^{n} \mathfrak{f}_{j} z_{j} \quad \text { and } \quad \underline{z}^{\dagger}=(\underline{z})^{\dagger}=\sum_{j=1}^{n} \mathfrak{f}_{j}^{\dagger} z_{j}^{c}
$$

where $n$ complex variables $z_{j}=x_{j}+i y_{j}$ have been introduced, with complex conjugates $z_{j}^{\mathrm{c}}=$ $x_{j}-i y_{j}, j=1, \ldots, n$. Finally, the Hermitean Dirac operators $\partial_{\underline{z}}$ and $\partial_{\underline{z}^{\dagger}}$ are obtained from the Euclidean Dirac operator $\partial_{\underline{x}}$ :

$$
\begin{aligned}
& \partial_{\underline{z}^{\dagger}}=\frac{1}{4}(1+i J)\left[\partial_{\underline{x}}\right]=\frac{1}{4}\left(\partial_{\underline{x}}+i \partial_{\underline{x} \mid}\right) \\
& \partial_{\underline{z}}=-\frac{1}{4}(1-i J)\left[\partial_{\underline{x}}\right]=-\frac{1}{4}\left(\partial_{\underline{x}}-i \partial_{\underline{x} \mid}\right)
\end{aligned}
$$

where also the so-called twisted Dirac operator arises:

$$
\partial_{\underline{X} \mid}=J\left[\partial_{\underline{X}}\right]=\sum_{j=1}^{n}\left(e_{j} \partial_{y_{j}}-e_{n+j} \partial_{x_{j}}\right)
$$

As for $\partial_{\underline{x}}$, a notion of (twisted) monogenicity may be associated in a natural way to $\partial_{\underline{X} \mid}$ as well. Passing to the Witt basis, the Hermitean Dirac operators are expressed as

$$
\partial_{\underline{z}}=\sum_{j=1}^{n} \mathfrak{f}_{j}^{\dagger} \partial_{z_{j}} \quad \text { and } \quad \partial_{\underline{z}^{\dagger}}=\left(\partial_{\underline{z}}\right)^{\dagger}=\sum_{j=1}^{n} \mathfrak{f}_{j} \partial_{z_{j}^{c}}
$$

involving the classical Cauchy-Riemann operators $\partial_{z_{j}^{c}}=\frac{1}{2}\left(\partial_{x_{j}}+i \partial_{y_{j}}\right)$ and their complex conjugates $\partial_{z_{j}}=\frac{1}{2}\left(\partial_{x_{j}}-i \partial_{y_{j}}\right)$ in the complex $z_{j}$-planes, $j=1, \ldots, n$. As a consequence of the isotropy of the Witt basis vectors, the Hermitean vector variables and Dirac operators are isotropic, i.e.

$$
(\underline{z})^{2}=\left(\underline{z}^{\dagger}\right)^{2}=0 \quad \text { and } \quad\left(\partial_{\underline{z}}\right)^{2}=\left(\partial_{\underline{z}^{\dagger}}\right)^{2}=0
$$

whence the Laplacian $\Delta_{2 n}=-\partial_{\underline{X}}^{2}=-\partial_{\underline{X} \mid}^{2}$ allows for the decomposition

$$
\Delta_{2 n}=4\left(\partial_{\underline{z}} \partial_{\underline{z}^{\dagger}}+\partial_{\underline{z}^{\dagger}} \partial_{\underline{z}}\right)=4\left(\partial_{\underline{z}}+\partial_{\underline{z}^{\dagger}}\right)^{2}
$$

while also

$$
\left(\underline{z}+\underline{z}^{\dagger}\right)^{2}=\underline{z} \underline{z}^{\dagger}+\underline{z}^{\dagger} \underline{z}=|\underline{z}|^{2}=\left|\underline{z}^{\dagger}\right|^{2}=|\underline{X}|^{2}=|\underline{X}|^{2}
$$

The central notion in Hermitean Clifford analysis is that of Hermitean monogenicity. A continuously differentiable function $\mathrm{g}$ on an open region $\mathrm{G}$ of $\mathbb{R}^{2 n} \cong \mathbb{C}^{n}$ with values in the complex 
Clifford algebra $\mathbb{C}_{2 n}$ is called (left) Hermitean monogenic (or $h$-monogenic for short) in $G$ if and only if it simultaneously is $\partial_{\underline{x}}-$ and $\left.\partial_{\underline{X}}\right|^{-m o n o g e n i c}$ in $G$, i.e. it satisfies in $G$ the system

$$
\partial_{\underline{x}} g=0=\partial_{\underline{x} \mid} g
$$

which is equivalent with the system

$$
\partial_{\underline{z}} g=0=\partial_{\underline{z}^{\dagger}} g
$$

Now the multivector functions in the Hermitean Clifford analysis setting are smooth functions defined in an open region $G$ of $\mathbb{R}^{2 n} \cong \mathbb{C}^{n}$ and taking their values in the Grassmann subspaces $\bigwedge_{2 \mathrm{n}}^{\mathrm{p}, \mathrm{q}}$. They thus take the form

$$
F^{p, q}\left(x_{1}, \ldots, x_{n}, y_{1}, \ldots, y_{n}\right)=\sum \phi_{j_{1}} \ldots j_{p} k_{1} \ldots k_{q} f_{j_{1}}^{\dagger} \wedge \ldots \wedge f_{j_{p}}^{\dagger} \wedge \mathfrak{f}_{k_{1}} \wedge \ldots \wedge \mathfrak{f}_{k_{q}}
$$

where the scalar functions $\phi_{j_{1} \ldots j_{p} k_{1} \ldots k_{q}}$ are assumed to be smooth functions in G. The space of these multivector functions is denoted by $\mathcal{E}^{p, q}(G)$, and we have

$$
\mathcal{E}^{\mathrm{r}}(\mathrm{G})=\bigoplus_{p+q=r} \mathcal{E}^{p, q}(\mathrm{G})
$$

Similarly to what was done for the Euclidean Dirac operator $\partial_{\underline{x}}$ (and holds for $\partial_{\underline{X} \mid}$ as well), also the Hermitean Dirac operators may be split into their scalar or "dot" part and their bivector or "wedge" part, leading to

$$
\begin{aligned}
\partial_{\underline{z}} \wedge & =\sum_{i=1}^{n} \partial_{z_{i}} f_{i}^{\dagger} \wedge \\
\partial_{\underline{z}} \bullet & =\sum_{i=1}^{n} \partial_{z_{i}} f_{i}^{\dagger} \bullet \\
\partial_{\underline{z}^{\dagger}} \wedge & =\sum_{i=1}^{n} \partial_{z_{i}^{c}} f_{i} \wedge \\
\partial_{\underline{z}^{\dagger}} \bullet & =\sum_{i=1}^{n} \partial_{z_{i}^{c}} f_{i} \bullet
\end{aligned}
$$

for which it thus holds that

$$
\partial_{\underline{z}} \wedge+\partial_{\underline{z}} \bullet=\partial_{\underline{z}}, \quad \partial_{\underline{z}^{\dagger}} \wedge+\partial_{\underline{z}^{\dagger}} \bullet=\partial_{\underline{z}^{\dagger}}
$$

These operators have a two-fold action on $\mathcal{E}^{p, q}(G)$ in the sense that they act on the multi-vector by means of the inner and outer product with Witt basis vectors, and at the same time on the function coefficients by partial differentiation. They enjoy the following properties, which can be obtained through direct calculation.

Property 2.2. The Hermitean Dirac dot and wedge operators are interrelated by complex conjugation as follows: 
(i) $\left(\partial_{\underline{z}} \wedge\right)^{c}=-\partial_{\underline{z}^{\dagger}} \wedge$

(ii) $\left(\partial_{\underline{z}} \bullet\right)^{c}=-\partial_{\underline{z}^{\dagger}} \bullet$

Property 2.3. The Hermitean Dirac dot and wedge operators act as follows on the spaces $\mathcal{E}^{\mathrm{p}, \mathrm{q}}$ :

(i) $\partial_{\underline{z}} \wedge: \mathcal{E}^{p, q} \longrightarrow \mathcal{E}^{p+1, q}$

(ii) $\partial_{\underline{z}} \bullet: \mathcal{E}^{p, q} \longrightarrow \mathcal{E}^{p, q-1}$

(iii) $\partial_{\underline{z}^{\dagger}} \wedge: \mathcal{E}^{p, q} \longrightarrow \mathcal{E}^{p, q+1}$

(iv) $\partial_{\underline{z}^{\dagger}} \bullet: \mathcal{E}^{p, q} \longrightarrow \mathcal{E}^{p-1, q}$

Property 2.4. The Hermitean Dirac dot and wedge operators are isotropic:

(i) $\left(\partial_{\underline{z}} \wedge\right)^{2}=\left(\partial_{\underline{z}} \bullet\right)^{2}=\left(\partial_{\underline{z}^{\dagger}} \wedge\right)^{2}=\left(\partial_{\underline{z}^{\dagger}} \bullet\right)^{2}=0$

and they show the following anticommutation relations:

(ii) $\left(\partial_{\underline{z}} \wedge\right)\left(\partial_{\underline{z}} \bullet\right)+\left(\partial_{\underline{z}} \bullet\right)\left(\partial_{\underline{z}} \wedge\right)=0$

(iii) $\left(\partial_{\underline{z}^{\dagger}} \wedge\right)\left(\partial_{\underline{z}^{\dagger}} \bullet\right)+\left(\partial_{\underline{z}^{\dagger}} \bullet\right)\left(\partial_{\underline{z}^{\dagger}} \wedge\right)=0$

(iv) $\left(\partial_{\underline{z}} \wedge\right)\left(\partial_{\underline{z}^{\dagger}} \wedge\right)+\left(\partial_{\underline{z}^{\dagger}} \wedge\right)\left(\partial_{\underline{z}} \wedge\right)=0$

$(\mathrm{v})\left(\partial_{\underline{z}} \bullet\right)\left(\partial_{\underline{z}^{\dagger}} \bullet\right)+\left(\partial_{\underline{z}^{\dagger}} \bullet\right)\left(\partial_{\underline{z}} \bullet\right)=0$

Property 2.5. Composition of the Hermitean Dirac dot and wedge operators yields the following actions on the spaces $\mathcal{E}^{p, q}$ :

(i) $\overline{\left(\partial_{\underline{z}} \wedge\right)\left(\partial_{\underline{z}} \bullet\right)}=-\left(\partial_{\underline{z}} \wedge\right)\left(\partial_{\underline{z}} \bullet\right)=\left(\partial_{\underline{z}} \bullet\right)\left(\partial_{\underline{z}} \wedge\right): \mathcal{E}^{p, q} \longrightarrow \mathcal{E}^{p+1, q-1}$

(ii) $\overline{\left(\partial_{\underline{z}^{\dagger}} \wedge\right)\left(\partial_{\underline{z}^{\dagger}} \bullet\right)}=-\left(\partial_{\underline{z}^{\dagger}} \wedge\right)\left(\partial_{\underline{z}^{\dagger}} \bullet\right)=\left(\partial_{\underline{z}^{\dagger}} \bullet\right)\left(\partial_{\underline{z}^{\dagger}} \wedge\right): \mathcal{E}^{p, q} \longrightarrow \mathcal{E}^{p-1, q+1}$

(iii) $\left(\partial_{\underline{z}} \wedge\right)\left(\partial_{\underline{z}^{\dagger}} \wedge\right)=-\left(\partial_{\underline{z}^{\dagger}} \wedge\right)\left(\partial_{\underline{z}} \wedge\right): \mathcal{E}^{p, q} \longrightarrow \mathcal{E}^{p+1, q+1}$

(iv) $\left(\partial_{\underline{z}} \bullet\right)\left(\partial_{\underline{z}^{\dagger}} \bullet\right)=-\left(\partial_{\underline{z}^{\dagger}} \bullet\right)\left(\partial_{\underline{z}} \bullet\right): \mathcal{E}^{p, q} \longrightarrow \mathcal{E}^{p-1, q-1}$

Property 2.6. The Hermitean Dirac dot and wedge operators establish a decomposition of the Laplacian in the following ways:

(i) $\left(\partial_{\underline{z}} \wedge\right)\left(\partial_{\underline{z}^{\dagger}} \bullet\right)+\left(\partial_{\underline{z}^{\dagger}} \bullet\right)\left(\partial_{\underline{z}} \wedge\right)=\frac{1}{8} \Delta_{2 n}$

(ii) $\left(\partial_{\underline{z}^{\dagger}} \wedge\right)\left(\partial_{\underline{z}} \bullet\right)+\left(\partial_{\underline{z}} \bullet\right)\left(\partial_{\underline{z}^{\dagger}} \wedge\right)=\frac{1}{8} \Delta_{2 n}$ 
Property 2.7. The Hermitean Dirac dot and wedge operators establish decompositions of the corresponding Euclidean ones as follows:

(i) $\left(\partial_{\underline{z}^{\dagger}} \wedge\right)-\left(\partial_{\underline{z}} \wedge\right)=\frac{1}{2} \partial_{\underline{x}} \wedge,\left(\partial_{\underline{z}^{\dagger}} \bullet\right)-\left(\partial_{\underline{z}} \bullet\right)=\frac{1}{2} \partial_{\underline{x}} \bullet$

(ii) $\left(\partial_{\underline{z}^{\dagger}} \wedge\right)+\left(\partial_{\underline{z}} \wedge\right)=\frac{i}{2} \partial_{\underline{X}} \wedge,\left(\partial_{\underline{z}^{\dagger}} \bullet\right)+\left(\partial_{\underline{z}} \bullet\right)=\frac{i}{2} \partial_{\underline{X}} \bullet$

whence they also decompose the actual Euclidean Dirac operators as follows:

(iii) $\left(\partial_{\underline{z}^{\dagger}} \wedge\right)-\left(\partial_{\underline{z}} \wedge\right)+\left(\partial_{\underline{z}^{\dagger}} \bullet\right)-\left(\partial_{\underline{z}} \bullet\right)=\frac{1}{2} \partial_{\underline{x}}$

(iv) $\left(\partial_{\underline{z}^{\dagger}} \wedge\right)+\left(\partial_{\underline{z}} \wedge\right)+\left(\partial_{\underline{z}^{\dagger}} \bullet\right)+\left(\partial_{\underline{z}} \bullet\right)=\frac{i}{2} \partial_{\underline{X}}$

Now, let us come back for a moment to the notion of Hermitean monogenicity for multivector functions. A multivector function $F^{p, q}$ is h-monogenic if and only if simultaneously $\partial_{z} F^{p, q}=$ $\left(\partial_{\underline{z}} \bullet+\partial_{\underline{z}} \wedge\right) F^{p, q}=0$ and $\partial_{\underline{z}^{\dagger}} F^{p, q}=\left(\partial_{\underline{z}^{\dagger}} \bullet+\partial_{\underline{z}^{\dagger}} \wedge\right) F^{p, q}=0$, which, due to Property 2.3. is equivalent with the system

$$
\left\{\partial_{\underline{z}} \bullet F^{p, q}=0, \partial_{\underline{z}} \wedge F^{p, q}=0, \partial_{\underline{z}^{\dagger}} \bullet F^{p, q}=0, \partial_{\underline{z}^{\dagger}} \wedge F^{p, q}=0\right\}
$$

In view of Property 2.7 we then obtain the following remarkable result.

Proposition 2.8. For a multivector function $F^{p, q}$ the notions of $\partial_{\underline{X}}$-monogenicity, $\left.\partial_{\underline{X}}\right|^{-m o n o g e n i c i t y ~}$ and Hermitean monogenicity coincide.

Remark 2.9. Obviously the system of equations describing Hermitean monogenicity will take particular forms according to the values of the functions considered. In [2] we have shown e.g. that, if the function takes its values in the subspace of spinor space corresponding to minimal or maximal degree of homogeneity, then Hermitean monogenicity reduces to (anti-)holomorphy for a function of several complex variables. In that sense Proposition 2.8 now reveals that one particular Grassmann cell $\bigwedge_{2 n}^{p, q}$ can not be considered as an appropriate value space to study Hermitean monogenicity, since in that case it coincides with Euclidean monogenicity. It remains an interesting problem to discover appropriate value spaces in order to see the Hermitean monogenicity system reduce to a significant system of differential equations. To that end we have investigated in [10, how the complex Clifford algebra $\mathbb{C}_{2 n}$ decomposes into subspaces leading to exact sequences for the multiplicative action of the Witt basis vectors.

\section{Differential forms: preliminaries}

There exists a vast literature on differential forms; in particular we refer to e.g. [19, 24, for real differential forms and to [20, 21] for complex differential forms. Here we will only recall the basic concepts needed. 
Let $\mathbb{R}^{m}$ be endowed with the standard Euclidean metric. Denoting by $\bigwedge^{r} \mathbb{R}^{m}$ the space of alternating (or skew multilinear) real valued $r$-forms $(0 \leq r \leq m)$, the Grassmann algebra or exterior algebra over $\mathbb{R}^{m}$ is the graded associative algebra

$$
\bigwedge \mathbb{R}^{m}=\bigoplus_{r=0}^{m} \bigwedge^{r} \mathbb{R}^{m}
$$

endowed with the exterior multiplication. A basis for $\bigwedge^{r} \mathbb{R}^{m}$ is obtained as follows. Let $\left\{d X^{1}, \ldots, d X^{m}\right\}$ be a basis for the dual space $\left(\mathbb{R}^{m}\right)^{*}$ of $\mathbb{R}^{m}$. If, as before, the set $A=\left\{i_{1}, \ldots, i_{r}\right\} \subset M=\{0,1, \ldots, m\}$ is ordered in the natural way, put

$$
d X^{A}=d X^{i_{1}} \wedge d X^{i_{2}} \wedge \ldots \wedge d X^{i_{r}}
$$

and $d X^{\emptyset}=1$. Then for each $r=0,1, \ldots, m$, the set $\left\{d X^{A}: A \subset M\right.$ and $\left.|A|=r\right\}$ is a basis for $\bigwedge^{\mathrm{r}} \mathbb{R}^{\mathrm{m}}$. Note that in particular

$$
d X^{i} \wedge d X^{i}=0, \quad i=0,1, \ldots, m
$$

and

$$
d X^{i} \wedge d X^{j}+d X^{j} \wedge d X^{i}=0, \quad 0 \leq i \neq j \leq m
$$

A smooth $r$-form in an open region $G$ of $\mathbb{R}^{m}$ is a map

$$
\omega^{r}: G \rightarrow \bigwedge^{r} \mathbb{R}^{m}, \quad X \mapsto \sum_{|A|=r} \omega_{A}^{r}\left(X_{1}, \ldots, X_{m}\right) d X^{A}
$$

where, for each $A, \omega_{A}^{r}$ is a smooth real valued function in $G$. We denote by $\bigwedge^{r}(G)$ the space of smooth $r$-forms in $\mathrm{G}$ and we put

$$
\bigwedge(\mathrm{G})=\bigoplus_{\mathrm{r}=0}^{\mathrm{m}} \bigwedge^{\mathrm{r}}(\mathrm{G})
$$

The projection operator from $\bigwedge(\mathrm{G})$ onto $\bigwedge^{\mathrm{r}}(\mathrm{G})$ is denoted by $[\cdot]^{\mathrm{r}}$. A fundamental linear operator on the space of smooth forms is the exterior derivative $d$. It is first defined as $d: \bigwedge^{r}(G) \rightarrow \bigwedge^{r+1}(G)$ $(r<m)$, by

$$
\omega^{r}=\sum_{|A|=r} \omega_{A}^{r} d X^{A} \longmapsto d \omega^{r}=\sum_{A} \sum_{j} \partial_{X_{j}} \omega_{A}^{r} d X^{j} \wedge d X^{A}
$$

a definition which is then extended to $\bigwedge(\mathrm{G})$ by linearity. A second fundamental linear operator on the space of smooth forms is the Hodge co-derivative $d^{*}$. For $A=\left\{i_{i}, \ldots, i_{r}\right\} \subset M$ we denote

$$
d X^{A \backslash\left\{i_{j}\right\}}=d X^{i_{1}} \wedge \ldots \wedge d X^{i_{j-1}} \wedge\left[d X^{i_{j}} \wedge\right] d X^{i_{j+1}} \wedge \ldots \wedge d X^{i_{r}}
$$

and in a first step we put

$$
d^{*}\left(\omega_{A}^{r} d X^{A}\right)=\sum_{j=1}^{r}(-1)^{j} \partial_{X_{i_{j}}} \omega_{A}^{r} d X^{A \backslash\left\{i_{j}\right\}}
$$


Then $\mathrm{d}^{*}$ is defined as $\mathrm{d}^{*}: \bigwedge^{r}(\mathrm{G}) \rightarrow \bigwedge^{r-1}(\mathrm{G})(\mathrm{r}>0)$, by

$$
\omega^{r}=\sum_{|A|=r} \omega_{A}^{r} d X^{A} \longmapsto d^{*}\left(\omega^{r}\right)=\sum_{|A|=r} d^{*}\left(\omega_{A}^{r} d X^{A}\right)
$$

and this definition again is extended to the whole of $\Lambda(G)$ by linearity. A smooth $r$-form $\omega^{r}$ in $G$ is called closed if and only if $d \omega^{r}=0$; it is called co-closed if and only if $d^{*} \omega^{r}=0$; and it is called harmonic (in the sense of Hodge) when it is at the same time closed and co-closed. Introducing the operator $\mathrm{D}=\mathrm{d}+\mathrm{d}^{*}$, a necessary and sufficient condition for a smooth $r$-form $\omega^{r}$ in $\mathrm{G}$ to be harmonic thus reads

$$
D \omega^{r}=\left(d+d^{*}\right) \omega^{r}=0 \Longleftrightarrow\left\{\begin{array}{l}
d \omega^{r}=0 \\
d^{*} \omega^{r}=0
\end{array}\right.
$$

The system (园) is called the Hodge-de Rham system. Note that if $\omega^{r}$ is harmonic in an open region $\mathrm{G}$ of $\mathbb{R}^{\mathrm{m}}$ then automatically $\omega^{\mathrm{r}}$ satisfies $\Delta_{\mathrm{m}} \omega^{\mathrm{r}}=0$ in $\mathrm{G}$, since

$$
D^{2}=\left(d+d^{*}\right)^{2}=d d^{*}+d^{*} d=-\Delta_{m}
$$

The converse, however, is not true.

The action of the operators $d$ and $d^{*}$ on differential forms is two-fold in the sense that they act on the form itself as well as on the function coefficients by partial differentiation. In order to make this double action explicit we introduce the following symbolic notations for the operators $d$ and $d^{*}$ :

$$
\begin{aligned}
d & =\sum_{j=1}^{m}\left(d X^{j} \wedge\right) \partial X_{j} \\
d^{*} & =\sum_{j=1}^{m}\left(d X^{j} \bullet\right) \partial X_{j}
\end{aligned}
$$

with

$$
d X^{j} \bullet d X^{A}=d X^{j} \bullet\left(d X^{i_{1}} \wedge \ldots \wedge d X^{i_{r}}\right)=\sum_{k=1}^{r}(-1)^{k} \delta_{j i_{k}} d X^{A \backslash\left\{i_{k}\right\}}
$$

In this last action we recognize the contraction operators $\left.\partial x_{j}\right\rfloor, j=1, \ldots m$, given by

$$
\left.\left.\partial_{X_{j}}\right\rfloor d X^{A}=\partial_{X_{j}}\right\rfloor\left(d X^{i_{1}} \wedge \ldots \wedge d X^{i_{r}}\right)=\sum_{k=1}^{r}(-1)^{k-1} \delta_{j i_{k}} d X^{A \backslash\left\{i_{k}\right\}}
$$

acting only on the basis elements of the differential form, and not on the function coefficients. Apparently the contraction operator $\left.\partial_{X_{j}}\right\rfloor$ coincides with the "inner product"-operator $d X^{j} \bullet$ up to a minus sign:

$$
\left.\partial_{X_{j}}\right\rfloor=\left(-d X^{j} \bullet\right), \quad j=0,1, \ldots, m
$$


However bear in mind that contractions are more fundamental than dot products. Indeed, they can be introduced independently of a scalar product, and their behaviour is invariant under diffeomorphisms, which is not the case for the dot product. We then indeed have for the operators $d$ and $\mathrm{d}^{*}$

$$
\begin{aligned}
\left(\sum_{j=1}^{m}\left(d X^{j} \wedge\right) \partial_{X_{j}}\right)\left(\sum_{|A|=r} \omega_{A}^{r} d X^{A}\right) & =\sum_{|A|=r} \sum_{j=1}^{m}\left(\partial_{X_{j}} \omega_{A}^{r}\right) d X^{j} \wedge d X^{A}=d \omega^{r} \\
\left(\sum_{j=1}^{m}\left(d X^{j} \bullet\right) \partial_{X_{j}}\right)\left(\sum_{|A|=r} \omega_{A}^{r} d X^{A}\right) & =\sum_{|A|=r} \sum_{k=1}^{r}(-1)^{k}\left(\partial_{X_{i_{k}}} \omega_{A}^{r}\right) d X^{A \backslash\left\{i_{k}\right\}}=d^{*} \omega^{r}
\end{aligned}
$$

At this moment we make the transition from the Euclidean to the Hermitean Clifford setting, which, as above, is established by the introduction of the complex structure J, forcing the dimension to be even: $m=2 n$. We may now also consider a twisted exterior derivative $\mathrm{d} \mid$ and a twisted co-derivative $d^{*} \mid$, satisfying the following identities.

Property 3.1. It holds that

(i) $\mathrm{dd}|+\mathrm{d}| \mathrm{d}=0=\left.\mathrm{d}^{*} \mathrm{~d}\right|^{*}+\left.\mathrm{d}\right|^{*} \mathrm{~d}^{*}=0$

(ii) $\left.d d\right|^{*}+\left.d\right|^{*} d=0=d^{*} d|+d| d^{*}=0$

Appropriate complex linear combinations of these real operators will give rise to complex exterior derivatives and co-derivatives, but we will first consider the traditional complex differential forms in $\mathbb{C}^{n}$ or in an open region $G$ of $\mathbb{C}^{n}$. We call $\bigwedge^{p, q}(G)$ the space of complex differential forms of bidegree $(p, q)$ in $G$; it contains elements $\omega^{p, q}$ of the form

$$
\omega^{p, q}=\sum_{|J|=p} \sum_{|K|=q} \omega_{J, K}\left(\underline{z}, \underline{z}^{\dagger}\right) d z_{J} \wedge d z_{K}^{c}
$$

where $\omega_{K, L}\left(z_{1}, \ldots, z_{n}, z_{1}^{c}, \ldots, z_{n}^{c}\right)$ are smooth functions in $G$ and

$$
\begin{aligned}
& \mathrm{d} z_{\mathrm{J}}=\mathrm{d} z_{\mathrm{j}_{1}} \wedge \ldots \wedge \mathrm{d} z_{\mathrm{j}_{\mathrm{p}}}, \quad \mathrm{j}_{1}<\mathrm{j}_{2}<\ldots<\mathrm{j}_{\mathrm{p}} \\
& \mathrm{d} z_{\mathrm{K}}^{\mathrm{c}}=\mathrm{d} z_{\mathrm{k}_{1}}^{\mathrm{c}} \wedge \ldots \wedge \mathrm{d} z_{\mathrm{k}_{\mathrm{q}}}^{\mathrm{c}}, \quad \mathrm{k}_{1}<\mathrm{k}_{2}<\ldots<\mathrm{k}_{\mathrm{p}}
\end{aligned}
$$

The traditional derivatives in this setting are $\partial, \partial^{c}, \partial^{*}$ and $\partial^{* c}$. They are defined as follows on a complex differential form of bidegree $(p, q)$, definition which is then extended by linearity to an 
arbitrary complex differential form:

$$
\begin{aligned}
\partial \omega^{p, q} & =\sum_{|J|=p} \sum_{|K|=q} \partial \omega_{J, K} \wedge d z_{J} \wedge d z_{K}^{c} \\
\partial^{c} \omega^{p, q} & =\sum_{|J|=p} \sum_{|K|=q} \partial^{c} \omega_{J, K} \wedge d z_{J} \wedge d z_{K}^{c} \\
\partial^{*} \omega^{p, q} & =\sum_{|J|=p} \sum_{|K|=q} \partial^{*}\left(\omega_{J, K} d z_{J} \wedge d z_{K}^{c}\right) \\
\partial^{* c} \omega^{p, q} & =\sum_{|J|=p} \sum_{|K|=q} \partial^{* c}\left(\omega_{J, K} d z_{J} \wedge d z_{K}^{c}\right)
\end{aligned}
$$

with

$$
\begin{aligned}
\partial \omega_{J, K} & =\sum_{i=1}^{n}\left(\partial_{z_{i}} \omega_{J, K}\right) d z_{i} \\
\partial^{c} \omega_{J, K} & =\sum_{i=1}^{n}\left(\partial_{z_{i}^{c}} \omega_{J, K}\right) d z_{i}^{c} \\
\partial^{*}\left(\omega_{J, K} d z_{J} \wedge d z_{K}^{c}\right) & =\sum_{i=1}^{n}\left(\partial_{z_{i}^{c}} \omega_{J, K}\right) d z_{i}^{c} \bullet\left(d z_{J} \wedge d z_{K}^{c}\right) \\
\partial^{* c}\left(\omega_{J, K} d z_{J} \wedge d z_{K}^{c}\right) & =\sum_{i=1}^{n}\left(\partial_{z_{i}} \omega_{J, K}\right) d z_{i} \bullet\left(d z_{J} \wedge d z_{K}^{c}\right)
\end{aligned}
$$

Here we have introduced, for $j=1, \ldots, n$, the not commonly used operators $d z_{j} \bullet$ and $d z_{j}^{c} \bullet$, which, via their Euclidean counterparts, are in fact complex contraction operators. We have indeed, for all $j=1, \ldots, n$, that

$$
\begin{aligned}
\mathrm{d} z_{\mathrm{j}} \bullet & \left.\left.\left.=\left(\mathrm{d} x_{\mathrm{j}}+i d y_{j}\right) \bullet=d x_{j} \bullet+i d y_{j} \bullet=-\left(\partial_{x_{j}}\right\rfloor+i \partial_{y_{j}}\right\rfloor\right)=-2 \partial_{z_{j}^{c}}\right\rfloor \\
\mathrm{d} z_{j}^{\mathrm{c}} \bullet & \left.\left.\left.=\left(\mathrm{d} x_{j}-i d y_{j}\right) \bullet=d x_{j} \bullet-i d y_{j} \bullet=-\left(\partial_{x_{j}}\right\rfloor-i \partial_{y_{j}}\right\rfloor\right)=-2 \partial_{z_{j}}\right\rfloor
\end{aligned}
$$

The four complex derivatives may thus be written symbolically as

$$
\begin{aligned}
\partial & =\sum_{i=1}^{n} \partial_{z_{i}} d z_{i} \wedge \\
\partial^{c} & =\sum_{i=1}^{n} \partial \partial_{z_{i}^{c}} d z_{i}^{c} \wedge \\
\partial^{*} & =\sum_{i=1}^{n} \partial \partial_{i} d z_{i}^{c} \bullet \\
\partial^{* c} & =\sum_{i=1}^{n} \partial_{z_{i}} d z_{i} \bullet
\end{aligned}
$$

where it is explicitly shown that $\partial$ and $\partial^{c}$ act with a wedge product and $\partial^{*}$ and $\partial^{* c}$ with a dot product or contraction. They enjoy the following properties. 
Property 3.2. The complex derivatives $\partial, \partial^{c}$, $\partial^{*}$ and $\partial^{* c}$ act as follows on the spaces $\bigwedge^{p, q}(G)$ of complex differential forms of bidegree $(p, q)$ in $G$ :

(i) $\partial: \bigwedge^{p, q}(G) \longrightarrow \bigwedge^{p+1, q}(G)$

(ii) $\partial^{c}: \bigwedge^{p, q}(G) \longrightarrow \bigwedge^{p, q+1}(G)$

(iii) $\partial^{*}: \bigwedge^{p, q}(G) \longrightarrow \bigwedge^{p-1, q}(G)$

(iv) $\partial^{* c}: \bigwedge^{p, q}(G) \longrightarrow \bigwedge^{p, q-1}(G)$

Property 3.3. The complex derivatives $\partial, \partial^{c}, \partial^{*}$ and $\partial^{* c}$ satisfy the Kähler identities

(i) $\partial \partial^{* c}+\partial^{* c} \partial=0=\partial^{*} \partial^{c}+\partial^{c} \partial^{*}$

(ii) $\partial \partial^{c}+\partial^{c} \partial=0=\partial^{*} \partial^{* c}+\partial^{* c} \partial^{*}$

(iii) $\partial \partial^{*}+\partial^{*} \partial=-\frac{1}{2} \Delta_{2 n}=\partial^{c} \partial^{* c}+\partial^{* c} \partial^{c}$

In a very similar way as the Hermitean variables and Dirac operators are linked to their Euclidean counterparts, the Kählerian derivatives $\partial, \partial^{c}, \partial^{*}$ and $\partial^{* c}$ are linked to the exterior derivative and co-derivative and their twisted analogues.

Property 3.4. It holds that

(i) $\partial^{c}+\partial=d, \partial^{c}-\partial=i d \mid$

(ii) $\partial^{*}+\partial^{* c}=d^{*}, \partial^{*}-\partial^{* c}=\left.i d\right|^{*}$

whence we may also write

(iii) $\partial^{c}=\frac{1}{2}(d+i d \mid), \partial=\frac{1}{2}(d-i d \mid)$

(iv) $\partial^{*}=\frac{1}{2}\left(d^{*}+\left.i d\right|^{*}\right), \partial^{* c}=\frac{1}{2}\left(d^{*}-\left.i d\right|^{*}\right)$

\section{Differential forms and multi-vector functions: an identi- fication}

In [6] it is shown how the world of real differential forms in an open region $G$ of $\mathbb{R}^{m}$ and the world of Clifford algebra valued multi-vector functions in $\mathrm{G}$ may be naturally identified. The fundamental identification, adapted to the Hermitean setting, reads

$$
e_{i} \longleftrightarrow d x^{i}, \quad e_{n+i} \longleftrightarrow d y^{i}, \quad i=1, \ldots, n
$$

resulting in the identifications listed in Table 1 . Note that we have listed here only a few of these identifications; for more details we refer the reader to 6 . 


\begin{tabular}{|c|c|}
\hline$d=\sum_{i=1}^{n}\left(d x^{i} \wedge\right) \partial_{x_{i}}+\left(d y^{i} \wedge\right) \partial_{y_{i}}$ & $\partial_{\underline{x}} \wedge=\sum_{i=1}^{n}\left(e_{i} \wedge\right) \partial_{x_{i}}+\left(e_{n+i} \wedge\right) \partial_{y_{i}}$ \\
\hline$d^{*}=\sum_{i=0}^{n}\left(d x^{i} \bullet\right) \partial_{x_{i}}+\left(d y^{i} \bullet\right) \partial_{y_{i}}$ & $\partial_{\underline{x}} \bullet=\sum_{i=1}^{n}\left(e_{i} \bullet\right) \partial_{x_{i}}+\left(e_{n+i} \bullet\right) \partial_{y_{i}}$ \\
\hline$d \mid=\sum_{i=1}^{n}\left(d x^{i} \wedge\right) \partial_{y_{i}}-\left(d y^{i} \wedge\right) \partial_{x_{i}}$ & $\partial_{\underline{X} \mid} \wedge=\sum_{i=1}^{n}\left(e_{i} \wedge\right) \partial_{y_{i}}-\left(e_{n+i} \wedge\right) \partial_{x_{i}}$ \\
\hline$\left.d\right|^{*}=\sum_{i=0}^{n}\left(d x^{i} \bullet\right) \partial_{y_{i}}-\left(d y^{i} \bullet\right) \partial_{x_{i}}$ & $\partial_{\underline{X} \mid} \bullet=\sum_{i=1}^{n}\left(e_{i} \bullet\right) \partial_{y_{i}}-\left(e_{n+i} \bullet\right) \partial_{x_{i}}$ \\
\hline
\end{tabular}

Table 1: Identification of the Euclidean Dirac operators

This identification is now further developed in the Hermitean setting. For the Witt basis vectors one explicitly obtains the identifications

$$
\begin{aligned}
& \mathfrak{f}_{j}^{\dagger} \wedge=-\frac{1}{2}\left(e_{j}+i e_{n+j}\right) \wedge=-\frac{1}{2}\left(e_{j} \wedge+i e_{n+j} \wedge\right) \\
& \mathfrak{f}_{j}^{\dagger} \bullet=-\frac{1}{2}\left(e_{j}+i e_{n+j}\right) \bullet=-\frac{1}{2}\left(e_{j} \bullet+i e_{n+j} \bullet\right) \longleftrightarrow-\frac{1}{2}\left(d x^{j} \wedge+i d y^{j} \wedge\right)=-\frac{1}{2}\left(d z_{j} \wedge\right) \\
& \mathfrak{f}_{j} \wedge=\frac{1}{2}\left(e_{j}-i e_{n+j}\right) \wedge=\frac{1}{2}\left(e_{j} \wedge-i e_{n+j} \wedge\right)=-\frac{1}{2}\left(d z_{j} \bullet\right)
\end{aligned}
$$

and

$$
\mathfrak{f}_{j} \bullet=\frac{1}{2}\left(e_{j}-i e_{n+j}\right) \bullet=\frac{1}{2}\left(e_{j} \bullet-i e_{n+j} \bullet\right) \longleftrightarrow \frac{1}{2}\left(d x^{j} \bullet-i d y^{j} \bullet\right)=\frac{1}{2}\left(d z_{j}^{c} \bullet\right)
$$

listed in Table 2. The so-called inflation operator, denoted · 7 , is introduced below.

\begin{tabular}{|c|c|}
\hline $\mathfrak{f}_{j}^{\dagger} \wedge$ & $\left.-\frac{1}{2}\left(\mathrm{~d} z_{j} \wedge\right)=\partial_{z_{j}^{c}}\right\rceil$ \\
\hline $\mathfrak{f}_{j}^{\dagger} \bullet$ & $\left.-\frac{1}{2}\left(\mathrm{~d} z_{j} \bullet\right)=\partial_{z_{j}^{c}}\right\rfloor$ \\
\hline $\mathfrak{f}_{j} \wedge$ & $\left.\frac{1}{2}\left(d z_{j}^{c} \wedge\right)=-\partial_{z_{j}}\right\rceil$ \\
\hline $\mathfrak{f}_{j} \bullet$ & $\left.\frac{1}{2}\left(d z_{j}^{c} \bullet\right)=-\partial_{z_{j}}\right\rfloor$ \\
\hline
\end{tabular}

Table 2: Identification of the Witt basis vectors 
In the same order of ideas one explicitly obtains for the Hermitean Dirac operators

$$
\begin{aligned}
\partial_{\underline{z}^{\dagger}} \wedge=\sum_{j=1}^{n} \partial_{z_{j}^{c}} f_{j} \wedge & \sum_{j=1}^{n} \partial_{z_{j}^{c}} \frac{1}{2}\left(d z_{j}^{c} \wedge\right)=\frac{1}{2}\left(\partial^{c} \wedge\right) \\
\partial_{\underline{z}^{\dagger}} \bullet=\sum_{j=1}^{n} \partial_{z_{j}^{c}} f_{j} \bullet & \sum_{j=1}^{n} \partial_{z_{j}^{c}} \frac{1}{2}\left(d z_{j}^{c} \bullet\right)=\frac{1}{2}\left(\partial^{*} \bullet\right) \\
\partial_{\underline{z}} \wedge=\sum_{j=1}^{n} \partial_{z_{j}} f_{j}^{\dagger} \wedge & \longleftrightarrow \sum_{j=1}^{n} \partial_{z_{j}}\left(-\frac{1}{2}\right)\left(d z_{j} \wedge\right)=\left(-\frac{1}{2}\right)(\partial \wedge) \\
\partial_{\underline{z}} \bullet=\sum_{j=1}^{n} \partial_{z_{j}} f_{j}^{\dagger} \bullet & \sum_{j=1}^{n} \partial_{z_{j}}\left(-\frac{1}{2}\right)\left(d z_{j} \bullet\right)=\left(-\frac{1}{2}\right)\left(\partial^{* c} \bullet\right)
\end{aligned}
$$

as summarized in Table 3 .

\begin{tabular}{|l|l|}
\hline$\partial_{\underline{z}^{\dagger}} \wedge$ & $\frac{1}{2}\left(\partial^{c} \wedge\right)$ \\
\hline$\partial_{\underline{z}^{\dagger}} \bullet$ & $\frac{1}{2}\left(\partial^{*} \bullet\right)$ \\
\hline$\partial_{\underline{z}} \wedge$ & $-\frac{1}{2}(\partial \wedge)$ \\
\hline$\partial_{\underline{z}} \bullet$ & $-\frac{1}{2}\left(\partial^{* c} \bullet\right)$ \\
\hline
\end{tabular}

Table 3: Identification of the Hermitean Dirac operators

Through these identifications it becomes clear that the properties of the Hermitean Dirac operators on multivector functions listed in Section 2 and those of the Kählerian differential operators on complex differential forms listed in Section 3 are two faces of the same coin. This also implies that it suffices to prove a property in only one of these two worlds, automatically gaining the similar property in the other. To give an example, Proposition 2.12 is transposed as follows.

Proposition 4.1. A $(p, q)$-form $\omega^{p, q} \in \bigwedge^{p, q}(G)$ is harmonic in an open region $G$ of $\mathbb{C}^{n}$, i.e. it satisfies the Hodge-de Rham system $\left\{d \omega^{p, q}=0, d^{*} \omega^{p, q}=0\right\}$, if and only if in $G$ it is Hermitean monogenic, i.e. it satisfies the system $\left\{\partial \omega^{p, q}=0, \partial^{c} \omega^{p, q}=0, \partial^{*} \omega^{p, q}=0, \partial^{* c} \omega^{p, q}=0\right\}$, which implies that for a $(p, q)$-form $\omega^{p, q} \in \bigwedge^{p, q}(G)$ the notions harmonic, twisted harmonic and Hermitean monogenic coincide.

Another nice illustration of this identification is procured by the Euler operators. The Hermitean Euler operators

$$
\begin{aligned}
& E_{\underline{z}}=\sum_{j=1}^{n} z_{j} \partial_{z_{j}}=2 \underline{z} \bullet \partial_{\underline{z}} \\
& E_{\underline{z}}^{\dagger}=\sum_{j=1}^{n} z_{j}^{c} \partial_{z_{j}^{c}}=2 \underline{z}^{\dagger} \bullet \partial_{\underline{z}^{\dagger}}
\end{aligned}
$$


have shown their importance in constructing the Fischer decomposition of homogeneous polynomials in terms of Hermitean monogenic polynomials and the corresponding Howe dual pair (see [16, 7]). They have a natural close connection with the traditional Euclidean Euler operators, since

$$
\begin{aligned}
& E_{\underline{z}}=\frac{1}{2} E_{\underline{X}}+\frac{i}{2} \underline{X} \bullet \partial \underline{\underline{X}} \mid \\
& E_{\underline{z}}^{\dagger}=\frac{1}{2} E_{\underline{X}}+\frac{i}{2} \underline{X} \mid \cdot \partial \underline{x}
\end{aligned}
$$

whence

$$
\begin{aligned}
& E_{\underline{z}}+E_{\underline{z}}^{\dagger}=\sum_{j=1}^{n}\left(x_{j} \partial_{x_{j}}+y_{j} \partial_{y_{j}}\right)=E_{\underline{x}}=E_{\underline{X} \mid}=-\underline{X} \bullet \partial \underline{x}=-\underline{X}\left|\cdot \partial_{\underline{x}}\right| \\
& E_{\underline{z}}-E_{\underline{z}}^{\dagger}=i \sum_{j=1}^{n}\left(-x_{j} \partial_{y_{j}}+y_{j} \partial_{x_{j}}\right)=i \underline{X} \bullet \partial_{\underline{x} \mid}=-i \underline{X} \mid \bullet \partial \underline{x}
\end{aligned}
$$

It thus becomes clear that the Hermitean Euler operators are mutually complex conjugated scalar operators; note that they have the same expression in both worlds. In the world of differential forms we now focus on the contraction operators associated to the Hermitean Euler operators. To that end recall that we tend to denote contraction of a differential form by means of a "dot", more specifically $\left.\partial_{x_{\alpha}}\right\rfloor=-d X_{\alpha} \bullet$, yielding

$$
\left.\left.\partial_{\underline{x}}\right\rfloor=\sum_{\alpha=1}^{m} e_{\alpha} \partial x_{\alpha}\right\rfloor=-\sum_{\alpha=1}^{m} e_{\alpha} d X_{\alpha} \bullet=-d \underline{X} \bullet
$$

and also

$$
\begin{array}{r}
\left.\left.\partial_{\underline{z}}\right\rfloor=\sum_{j=1}^{n} f_{j}^{\dagger} \partial_{z_{j}}\right\rfloor=\sum_{j=1}^{n} f_{j}^{\dagger}\left(-\frac{1}{2} d z_{j}^{c} \bullet\right)=-\frac{1}{2} d \underline{z^{\dagger}} \bullet \\
\left.\left.\partial_{\underline{z}^{\dagger}}\right\rfloor=\sum_{j=1}^{n} f_{j} \partial_{z_{j}^{c}}\right\rfloor=\sum_{j=1}^{n} f_{j}\left(-\frac{1}{2} d z_{j} \bullet\right)=-\frac{1}{2} d \underline{z} \bullet
\end{array}
$$

For the contracted Hermitean Euler operators we then obtain

$$
\begin{aligned}
& \left.\left.\left.\left.E_{\underline{z}}\right\rfloor=\sum_{j=1}^{n} z_{j} \partial_{z_{j}}\right\rfloor=\left(-\frac{1}{2}\right) \sum_{j=1}^{n} z_{j}\left(d z_{j}^{c} \bullet\right) \quad \text { or } \quad E_{\underline{z}}\right\rfloor=2 \underline{z} \bullet \partial_{\underline{z}}\right\rfloor=-\underline{z} \bullet d \underline{z}^{\dagger} \bullet \\
& \left.\left.\left.\left.E_{\underline{z}}^{\dagger}\right\rfloor=\sum_{j=1}^{n} z_{j}^{c} \partial_{z_{j}^{c}}\right\rfloor=\left(-\frac{1}{2}\right) \sum_{j=1}^{n} z_{j}^{c}\left(d z_{j} \bullet\right) \quad \text { or } \quad E_{\underline{z}}^{\dagger}\right\rfloor=2 \underline{z}^{\dagger} \bullet \partial_{\underline{z}^{\dagger}}\right\rfloor=-\underline{z}^{\dagger} \bullet \mathrm{d} \underline{z} \bullet
\end{aligned}
$$

We could as well, for symmetry's sake, have introduced a so-called inflation operator (see [6]), denoted by a "wedge", i.e. $\left.\partial_{x_{\alpha}}\right\rceil=-d X_{\alpha} \wedge$, yielding

$$
\left.\partial \underline{\mathbf{X}}\rceil=\sum_{\alpha=1}^{m} e_{\alpha} \partial x_{\alpha}\right\rceil=-\sum_{\alpha=1}^{m} e_{\alpha} d X_{\alpha} \wedge=-d \underline{X} \wedge
$$


and

$$
\begin{array}{r}
\left.\left.\partial_{\underline{z}}\right\rceil=\sum_{j=1}^{n} f_{j}^{\dagger} \partial_{z_{j}}\right\rceil=\sum_{j=1}^{n} f_{j}^{\dagger}\left(-\frac{1}{2} d z_{j}^{c} \wedge\right)=-\frac{1}{2} d \underline{z}^{\dagger} \wedge \\
\left.\left.\partial_{\underline{z}^{\dagger}}\right\rceil=\sum_{j=1}^{n} f_{j} \partial_{z_{j}^{c}}\right\rceil=\sum_{j=1}^{n} \mathfrak{f}_{j}\left(-\frac{1}{2} d z_{j} \wedge\right)=-\frac{1}{2} d \underline{z} \wedge
\end{array}
$$

Note that the above notations $\left.\partial_{z_{j}}\right\rceil$ and $\left.\partial_{z_{j}^{c}}\right\rceil$ where already used in Table 2 . This leads to

$$
\begin{aligned}
& \left.\left.\left.\left.E_{\underline{z}}\right\rceil=\sum_{j=1}^{n} z_{j} \partial_{z_{j}}\right\rceil=\left(-\frac{1}{2}\right) \sum_{j=1}^{n} z_{j}\left(d z_{j}^{c} \wedge\right) \quad \text { or } \quad E_{\underline{z}}\right\rceil=2 \underline{z} \bullet \partial_{\underline{z}}\right\rceil=-\underline{z} \bullet d \underline{z}^{\dagger} \wedge \\
& \left.\left.\left.\left.E_{\underline{z}}^{\dagger}\right\rceil=\sum_{j=1}^{n} z_{j}^{c} \partial_{z_{j}^{c}}\right\rceil=\left(-\frac{1}{2}\right) \sum_{j=1}^{n} z_{j}^{c}\left(d z_{j} \wedge\right) \quad \text { or } \quad E_{\underline{z}}^{\dagger}\right\rceil=2 \underline{z}^{\dagger} \bullet \partial_{\underline{z}^{\dagger}}\right\rceil=-\underline{z}^{\dagger} \bullet \mathrm{d} \underline{z} \wedge
\end{aligned}
$$

These four contracted and inflated Hermitean Euler operators enjoy the properties summarized in the following two propositions.

Proposition 4.2. One has

(i) $\left.\left.\left.E_{\underline{z}}\right\rfloor+E_{\underline{z}}^{\dagger}\right\rfloor=E_{\underline{X}}\right\rfloor=\underline{X} \bullet \mathrm{d} \underline{X} \bullet$

(ii) $\left.\left.\left.E_{\underline{z}}\right\rceil+E_{\underline{z}}^{\dagger}\right\rceil=E_{\underline{X}}\right\rceil=\underline{X} \bullet d \underline{X} \wedge$

(iii) $\left.\left.E_{\underline{z}}\right\rfloor-E_{z}^{\dagger}\right\rfloor=i \underline{X}|\bullet d \underline{X} \bullet=-i \underline{X} \bullet d \underline{X}| \bullet$

(iv) $\left.\left.E_{\underline{z}}\right\rceil-E_{z}^{\dagger}\right\rceil=i \underline{X}|\bullet d \underline{X} \wedge=-i \underline{X} \bullet d \underline{X}| \wedge$

(v) $\left.\left.E_{z}\right\rfloor+E_{z}\right\rceil=\left(-\frac{1}{2}\right) \sum_{j=1}^{n} z_{j} d z_{j}^{c}=-\underline{z} \bullet d \underline{z}^{\dagger}$

(vi) $\left.\left.E_{z}^{\dagger}\right\rfloor+E_{z}^{\dagger}\right\rceil=\left(-\frac{1}{2}\right) \sum_{j=1}^{n} z_{j}^{c} d z_{j}=-\underline{z}^{\dagger} \bullet d \underline{z}$

Proposition 4.3. One has

(i) $\left.\left.\left.\left(E_{z}\right\rfloor+E_{z}^{\dagger}\right\rfloor\right)^{2}=\left(E_{\underline{x}}\right\rfloor\right)^{2}=0$

(ii) $\left.\left.\left.\left(E_{z}\right\rceil+E_{z}^{\dagger}\right\rceil\right)^{2}=\left(E_{\underline{X}}\right\rceil\right)^{2}=0$

(iii) $\left.\left.\left(E_{z}\right\rfloor+E_{z}\right\rceil\right)^{2}=0$

(iv) $\left.\left.\left(E_{z}^{\dagger}\right\rfloor+E_{z}^{\dagger}\right\rceil\right)^{2}=0$

(v) $\left.\left.\left.\left.\left.\left.\left.\left.\left(E_{z}\right\rfloor+E_{z}\right\rceil\right)\left(E_{z}^{\dagger}\right\rfloor+E_{z}^{\dagger}\right\rceil\right)+\left(E_{z}^{\dagger}\right\rfloor+E_{z}^{\dagger}\right\rceil\right)\left(E_{z}\right\rfloor+E_{z}\right\rceil\right)=-\left|\underline{z}^{2}\right|$ 
(vi) $\left.\left.\left.\left.\left.\left.\left.\left.\left(E_{z}\right\rfloor+E_{z}^{\dagger}\right\rfloor\right)\left(E_{z}\right\rceil+E_{z}^{\dagger}\right\rceil\right)+\left(E_{z}\right\rceil+E_{z}^{\dagger}\right\rceil\right)\left(E_{z}\right\rfloor+E_{z}^{\dagger}\right\rfloor\right)=-\left|\underline{z}^{2}\right|$

These properties may be proven by direct calculation, but things become more transparent after identification in the multivector setting; to that end we look at the analogues of the operators involved, given by

$$
\begin{aligned}
& \left.\mathrm{E}_{\underline{z}}\right\rfloor=\left(-\frac{1}{2}\right) \sum_{j=1}^{n} z_{j}\left(\mathrm{~d} z_{j}^{c} \bullet\right) \longleftrightarrow\left(-\frac{1}{2}\right) \sum_{j=1}^{n} z_{j}\left(2 \mathfrak{f}_{j} \bullet\right)=-\underline{z} \bullet \\
& \left.\mathrm{E}_{\underline{z}}^{\dagger}\right\rfloor=\left(-\frac{1}{2}\right) \sum_{j=1}^{n} z_{j}^{c}\left(\mathrm{~d} z_{j} \bullet\right) \longleftrightarrow\left(-\frac{1}{2}\right) \sum_{j=1}^{n} z_{j}^{c}\left(-2 \mathfrak{f}_{j}^{\dagger} \bullet\right)=\underline{z}^{\dagger} \bullet \\
& \left.\mathrm{E}_{\underline{z}}\right\rceil=\left(-\frac{1}{2}\right) \sum_{j=1}^{n} z_{j}\left(\mathrm{~d} z_{j}^{c} \wedge\right) \longleftrightarrow\left(-\frac{1}{2}\right) \sum_{j=1}^{n} z_{j}\left(2 \mathfrak{f}_{j} \wedge\right)=-\underline{z} \wedge \\
& \left.\mathrm{E}_{\underline{z}}^{\dagger}\right\rceil=\left(-\frac{1}{2}\right) \sum_{j=1}^{n} z_{j}^{c}\left(\mathrm{~d} z_{j} \wedge\right) \longleftrightarrow\left(-\frac{1}{2}\right) \sum_{j=1}^{n} z_{j}^{c}\left(-2 \mathfrak{f}_{j}^{\dagger} \wedge\right)=\underline{z}^{\dagger} \wedge
\end{aligned}
$$

Propositions 4.2 and 4.3 now take a rather trivial form and are easily proven (see also [6]), as may be observed from their reformulation in the propositions below.

Proposition 4.4. One has

(i) $(-\underline{z} \bullet)+\left(\underline{z}^{\dagger} \bullet\right)=-\underline{X} \bullet$

(ii) $(-\underline{z} \wedge)+\left(\underline{z}^{\dagger} \wedge\right)=-\underline{X} \wedge$

(iii) $(-\underline{z} \bullet)-\left(\underline{z}^{\dagger} \bullet\right)=-\underline{i} \underline{X} \bullet$

(iv) $(-\underline{z} \wedge)-\left(\underline{z}^{\dagger} \wedge\right)=-\underline{i} \underline{X} \mid \wedge$

(v) $(-\underline{z} \bullet)+(-\underline{z} \wedge)=-\underline{z}$

(vi) $\left(\underline{z}^{\dagger} \bullet\right)+\left(\underline{z}^{\dagger} \wedge\right)=\underline{z}^{\dagger}$

Proposition 4.5. One has

(i) $(-\underline{X} \bullet)(\underline{X} \bullet)=0$

(ii) $(-\underline{X} \wedge)(\underline{X} \wedge)=0$

(iii) $(-\underline{z} \bullet-\underline{z} \wedge)^{2}=(-\underline{z})^{2}=0$

(iv) $\left(\underline{z}^{\dagger} \bullet+\underline{z}^{\dagger} \wedge\right)^{2}=\left(\underline{z}^{\dagger}\right)^{2}=0$

(v) $(-\underline{z})\left(\underline{z}^{\dagger}\right)+\left(\underline{z}^{\dagger}\right)(-\underline{z})=-\left|\underline{z}^{2}\right|$

(vi) $\left(-\underline{z} \bullet+\underline{z}^{\dagger} \bullet\right)\left(-\underline{z} \wedge+\underline{z}^{\dagger} \wedge\right)+\left(-\underline{z} \wedge+\underline{z}^{\dagger} \wedge\right)\left(-\underline{z} \bullet+\underline{z}^{\dagger} \bullet\right)=-\left|\underline{z}^{2}\right|$ 
In the same order of ideas, starting from the operators $d$ and $d^{*}$, we introduce the contraction and inflation operators

$$
\begin{aligned}
d\rfloor & \left.=\sum_{j=1}^{m}\left(d X^{j} \wedge\right) \partial_{X_{j}}\right\rfloor=\sum_{j=1}^{m}\left(d X^{j} \wedge\right)\left(-d X^{j} \bullet\right) \\
\left.d^{*}\right\rceil & \left.=\sum_{j=1}^{m}\left(d X^{j} \bullet\right) \partial_{X_{j}}\right\rceil=\sum_{j=1}^{m}\left(d X^{j} \bullet\right)\left(-d X^{j} \wedge\right)
\end{aligned}
$$

The operators $d\rfloor$ and $\left.d^{*}\right\rceil$ have $\mathcal{E}^{r}(\Omega)$ as an eigenspace since

$$
\left.\mathrm{d}\rfloor \omega^{r}=r \omega^{r} \quad \text { and } \quad d^{*}\right\rceil \omega^{r}=(m-r) \omega^{r}
$$

In other words: they measure the order of a differential form. They are sometimes called fermionic Euler operators. In the Clifford analysis setting they read

$$
\left.\left.\partial_{\underline{x}} \wedge\right\rfloor=\sum_{j=1}^{m}\left(e_{j} \wedge\right)\left(-e_{j} \bullet\right) \quad \text { and } \quad \partial_{\underline{x}} \bullet\right\rceil=\sum_{j=1}^{m}\left(e_{j} \bullet\right)\left(-e_{j} \wedge\right)
$$

for which it indeed holds that

$$
\left.\partial_{\underline{X}} \wedge J F_{r}=r F_{r} \quad \text { and } \quad \partial_{\underline{X}} \bullet\right\rceil F_{r}=(m-r) F_{r}
$$

Note that $\left.\left.d\rceil, d^{*}\right\rfloor, \partial_{\underline{x}} \wedge\right\rceil$ and $\left.\partial_{\underline{x}} \bullet\right\rfloor$ are zero operators. The same can be done now with the Kählerian derivatives, leading to

$$
\begin{aligned}
\partial\rfloor & =\left(-\frac{1}{2}\right) \sum_{j=1}^{n} \mathrm{~d} z_{j} \wedge \mathrm{d} z_{j}^{\mathrm{c}} \bullet \\
\left.\partial^{\mathrm{c}}\right\rfloor & =\left(-\frac{1}{2}\right) \sum_{j=1}^{\mathrm{n}} \mathrm{d} z_{j}^{\mathrm{c}} \wedge \mathrm{d} z_{j} \bullet \\
\left.\partial^{*}\right\rfloor & =\left(-\frac{1}{2}\right) \sum_{j=1}^{\mathrm{n}} \mathrm{d} z_{j}^{\mathrm{c}} \bullet \mathrm{d} z_{j} \bullet \\
\left.\partial^{* c}\right\rfloor & =\left(-\frac{1}{2}\right) \sum_{j=1}^{n} \mathrm{~d} z_{j} \bullet \mathrm{d} z_{j}^{\mathrm{c}} \bullet
\end{aligned}
$$

and

$$
\begin{aligned}
\partial\rceil & =\left(-\frac{1}{2}\right) \sum_{j=1}^{n} \mathrm{~d} z_{j} \wedge \mathrm{d} z_{j}^{\mathrm{c}} \wedge \\
\left.\partial^{\mathrm{c}}\right\rceil & =\left(-\frac{1}{2}\right) \sum_{j=1}^{\mathrm{n}} \mathrm{d} z_{j}^{\mathrm{c}} \wedge \mathrm{d} z_{j} \wedge \\
\left.\partial^{*}\right\rceil & =\left(-\frac{1}{2}\right) \sum_{j=1}^{\mathrm{n}} \mathrm{d} z_{j}^{\mathrm{c}} \bullet \mathrm{d} z_{j} \wedge \\
\left.\partial^{* \mathrm{c}}\right\rceil & =\left(-\frac{1}{2}\right) \sum_{j=1}^{n} \mathrm{~d} z_{j} \bullet \mathrm{d} z_{j}^{\mathrm{c}} \wedge
\end{aligned}
$$


while their Hermitean multivector analogues are given by

$$
\begin{aligned}
\left.\partial_{\underline{z}} \wedge\right\rfloor & =-\sum_{j=1}^{n} f_{j}^{\dagger} \wedge f_{j} \bullet \\
\left.\partial_{\underline{z}^{\dagger}} \wedge\right\rfloor & =\sum_{j=1}^{n} f_{j} \wedge f_{j}^{\dagger} \bullet \\
\left.\partial_{\underline{z}^{\dagger}} \bullet\right\rfloor & =\sum_{j=1}^{n} f_{j} \bullet f_{j}^{\dagger} \bullet \\
\left.\partial_{\underline{z}} \bullet\right\rfloor & =-\sum_{j=1}^{n} f_{j}^{\dagger} \bullet f_{j} \bullet
\end{aligned}
$$

and by

$$
\begin{aligned}
\left.\partial_{\underline{z}} \wedge\right\rceil & =-\sum_{j=1}^{n} f_{j}^{\dagger} \wedge \mathfrak{f}_{j} \wedge \\
\left.\partial_{\underline{z}^{\dagger}} \wedge\right\rceil & =\sum_{j=1}^{n} f_{j} \wedge f_{j}^{\dagger} \wedge \\
\left.\partial_{\underline{z}^{\dagger}} \bullet\right\rceil & =\sum_{j=1}^{n} \mathfrak{f}_{j} \bullet f_{j}^{\dagger} \wedge \\
\left.\partial_{\underline{z}} \bullet\right\rceil & =-\sum_{j=1}^{n} f_{j}^{\dagger} \bullet f_{j} \wedge
\end{aligned}
$$

The spaces $\mathcal{E}^{p, q}$ of smooth vector functions of bidegree $(p, q)$ are eigenspaces of the operators $\left.\left.\left.\partial_{\underline{z}} \wedge\right\rfloor, \partial_{\underline{z}^{\dagger}} \wedge\right\rfloor, \partial_{\underline{z}^{\dagger}} \bullet\right\rfloor$ and $\left.\partial_{\underline{z}} \bullet\right\rfloor$. More precisely we have the following.

Proposition 4.6. For $\mathrm{F}^{\mathrm{p}, \mathrm{q}} \in \mathcal{E}^{\mathrm{p}, \mathrm{q}}$, one has

(i) $\left.\left(\partial_{\underline{z}} \wedge\right\rfloor\right) F^{p, q}=\left(-\frac{p}{2}\right) F^{p, q}$

(ii) $\left.\left(\partial_{\underline{z}^{\dagger}} \wedge\right\rfloor\right) F^{p, q}=\left(\frac{q}{2}\right) F^{p, q}$

(iii) $\left.\left(\partial_{\underline{z}^{\dagger}} \bullet\right\rceil\right) F^{p, q}=\left(\frac{n-p}{2}\right) F^{p, q}$

(iv) $\left.\left(\partial_{\underline{z}} \bullet\right\urcorner\right) F^{p, q}=\left(\frac{-n+q}{2}\right) F^{p, q}$

Note that, by similarity, the same eigenvalue equations hold for the operators $\left.\left.\partial\rfloor, \partial^{c}\right\rfloor, \partial^{*}\right\rceil$ and $\left.\partial^{* c}\right\rceil$. Moreover observe that the eigenvalue equations for the operators $\left.d\right\rfloor$ or $\left.\partial \underline{x} \wedge\right\rfloor$ and $\left.d^{*}\right\rceil$ or 
$\partial_{\underline{x}} \bullet 1$ are refined by the ones of Proposition 4.6, and may be recovered from them:

$$
\begin{aligned}
\left(\partial_{\underline{x}} \wedge J\right) F^{p, q} & \left.\left.=2\left(\partial_{\underline{z}^{\dagger}} \wedge\right\rfloor-\partial_{\underline{z}} \wedge\right\rfloor\right) F^{p, q}=2\left(\frac{q}{2}+\frac{p}{2}\right) F^{p, q} \\
& =(p+q) F^{p, q} \\
\left.\left(\partial_{\underline{x}} \bullet\right\rceil\right) F^{p, q} & \left.\left.=2\left(\partial_{\underline{z}^{\dagger}} \bullet\right\rceil-\partial_{\underline{z}} \bullet\right\rceil\right) F^{p, q}=2\left(\frac{n-p}{2}-\frac{-n+q}{2}\right) F^{p, q} \\
& =(2 n-(p+q)) F^{p, q}
\end{aligned}
$$

Furthermore, it may be verified that $\left.\partial_{\underline{x}} \wedge\right\rceil$ and $\left.\partial_{\underline{x}} \bullet\right\rfloor$ indeed are zero operators:

$$
\begin{aligned}
& \left.\left.\left.\partial_{\underline{x}} \wedge\right\rceil=2\left(\partial_{\underline{z}^{\dagger}} \wedge\right\rceil-\partial_{\underline{z}} \wedge\right\rceil\right)=2\left(\sum_{j=1}^{n} \mathfrak{f}_{j} \wedge \mathfrak{f}_{j}^{\dagger} \wedge+\sum_{j=1}^{n} \mathfrak{f}_{j}^{\dagger} \wedge \mathfrak{f}_{j} \wedge\right)=0 \\
& \left.\left.\left.\partial_{\underline{x}} \bullet\right\rfloor=2\left(\partial_{\underline{z}^{\dagger}} \bullet\right\rfloor-\partial_{\underline{z}} \bullet\right\rfloor\right)=2\left(\sum_{j=1}^{n} \mathfrak{f}_{j} \bullet \mathfrak{f}_{j}^{\dagger} \bullet+\sum_{j=1}^{n} \mathfrak{f}_{j}^{\dagger} \bullet \mathfrak{f}_{j} \bullet\right)=0
\end{aligned}
$$

Finally, also the original expressions for $\left.\partial_{\underline{x}} \wedge\right\rfloor$ and $\left.\partial_{\underline{x}} \bullet\right]$ as obtained in $[6]$ may be recovered:

$$
\begin{aligned}
\left.\partial_{\underline{x}} \wedge\right\rfloor & \left.=2\left(\partial_{\underline{z}^{\dagger}} \wedge\right\rfloor-\partial_{\underline{z}} \wedge J\right)=2\left(\sum_{j=1}^{n} \mathfrak{f}_{j} \wedge \mathfrak{f}_{j}^{\dagger} \bullet+\sum_{j=1}^{n} f_{j}^{\dagger} \wedge \mathfrak{f}_{j} \bullet\right) \\
& =-\sum_{j=1}^{n} e_{j} \wedge e_{j} \bullet+e_{n+j} \wedge e_{n+j} \bullet=-\sum_{\alpha=1}^{2 n} e_{\alpha} \wedge e_{\alpha} \bullet \\
\left.\partial_{\underline{x}} \bullet\right\rceil & \left.\left.=2\left(\partial_{\underline{z}^{\dagger}} \bullet\right\rceil-\partial_{\underline{z}} \bullet\right\rceil\right)=2\left(\sum_{j=1}^{n} \mathfrak{f}_{j} \bullet \mathfrak{f}_{j}^{\dagger} \wedge+\sum_{j=1}^{n} \mathfrak{f}_{j}^{\dagger} \bullet \mathfrak{f}_{j} \wedge\right) \\
& =-\sum_{j=1}^{n} e_{j} \bullet e_{j} \wedge+e_{n+j} \bullet e_{n+j} \wedge=-\sum_{\alpha=1}^{2 n} e_{\alpha} \bullet e_{\alpha} \wedge
\end{aligned}
$$

We shall encounter the operators $\left.\partial_{\underline{z}} \wedge\right\rceil$ and $\left.\partial_{\underline{z}} \bullet\right\rfloor$ again in the next section in a different context.

\section{The Kählerian metric}

We will now use known results from Kählerian geometry, however restricted to the flat Kählerian manifold $\mathbb{C}^{n}$, and transpose them to obtain results, not yet known in the Hermitean Clifford analysis setting. Our guides are [21, 20]. Each Kählerian metric induces a fundamental form $\Omega$, which is a 2-form derived from the corresponding Kähler potential $\mathrm{U}$, i.e.

$$
\Omega \wedge=\frac{i}{2} \partial \partial^{c} \mathrm{u}
$$

The potential of the flat metric or the canonical Hermitean metric is given by

$$
\mathrm{U}=\frac{1}{2}|\underline{z}|^{2}=\frac{1}{2}\left|\underline{z}^{\dagger}\right|^{2}=\frac{1}{2} \sum_{i=1}^{n} z_{\mathrm{i}} z_{\mathrm{i}}^{\mathrm{c}}=\underline{z} \bullet \underline{z}^{\dagger}=\frac{1}{2}\left(\underline{z z^{\dagger}}+\underline{z}^{\dagger} \underline{z}\right)=\frac{1}{2}|\underline{X}|^{2}=\frac{1}{2}|\underline{X}|^{2}
$$


yielding the flat fundamental form

$$
\begin{aligned}
\Omega \wedge & =\frac{i}{2}\left(-2 \partial_{\underline{z}} \wedge\right)\left(2 \partial_{\underline{z}^{\dagger}} \wedge\right)|\underline{z}|^{2}=(-2 i)\left(\sum_{j=1}^{n} \partial_{z_{j}} f_{j}^{\dagger} \wedge\right)\left(\sum_{k=1}^{n} \partial_{z_{k}^{c}} \mathfrak{f}_{k} \wedge\right)|\underline{z}|^{2} \\
& \left.=(-2 i) \sum_{j=1}^{n} f_{j}^{\dagger} \wedge \mathfrak{f}_{j} \wedge=2 i \partial_{\underline{z}} \wedge\right\rceil
\end{aligned}
$$

or, in terms of the original basis vectors,

$$
\Omega \wedge=\sum_{j=1}^{n} e_{j} \wedge e_{n+j} \wedge
$$

Introducing the so-called spin-Euler operator, which is a parabivector valued multiplicative constant, i.e. the sum of a scalar and a bivector,

$$
\beta=\sum_{j=1}^{n} \mathfrak{f}_{j}^{\dagger} \mathfrak{f}_{j}=\sum_{j=1}^{n}\left(\mathfrak{f}_{j}^{\dagger} \bullet \mathfrak{f}_{j}+\mathfrak{f}_{j}^{\dagger} \wedge \mathfrak{f}_{j}\right)=\frac{n}{2}+\sum_{j=1}^{n} \mathfrak{f}_{j}^{\dagger} \wedge \mathfrak{f}_{j}
$$

we find that the fundamental form appears as the bivector part of that spin-Euler operator, meaning that we may write

$$
\beta=\frac{n}{2}+\frac{i}{2} \Omega
$$

Its complex conjugate is then given by

$$
\beta^{c}=\sum_{j=1}^{n} \mathfrak{f}_{j} \mathfrak{f}_{j}^{\dagger}=\sum_{j=1}^{n}\left(\mathfrak{f}_{j} \bullet \mathfrak{f}_{j}^{\dagger}+\mathfrak{f}_{j} \wedge \mathfrak{f}_{j}^{\dagger}\right)=\frac{n}{2}-\sum_{j=1}^{n} \mathfrak{f}_{j}^{\dagger} \wedge \mathfrak{f}_{j}=\frac{n}{2}-\frac{i}{2} \Omega
$$

Usually, one also introduces the associated fundamental form

$$
\omega=\frac{1}{2 i} \Omega=\frac{n}{2}-\beta=\beta^{c}-\frac{n}{2}=\sum_{j=1}^{n} \mathfrak{f}_{j} \wedge \mathfrak{f}_{j}^{\dagger}
$$

For the sake of completeness we recall the following intertwining relations of the spin-Euler operator and its complex conjugate with the Witt basis vectors; for more of these intertwining relations we refer to 9 .

Proposition 5.1. One has

(i) $\left[\mathfrak{f}_{k}, \beta\right]=\mathfrak{f}_{k},\left[\mathfrak{f}_{k}^{\dagger}, \beta\right]=-\mathfrak{f}_{k}^{\dagger}$

(ii) $\left[\mathfrak{f}_{k}^{\dagger}, \beta^{c}\right]=\mathfrak{f}_{k}^{\dagger},\left[\mathfrak{f}_{k}, \beta^{c}\right]=-\mathfrak{f}_{k}$

An important operator in Kähler geometry is the so-called L-operator, which is defined by means of the fundamental form. 
Definition 5.2. The L-operator is defined as $\mathrm{L}: \mathcal{E}^{\mathrm{p}, \mathrm{q}} \longrightarrow \mathcal{E}^{\mathrm{p}+1, \mathrm{q}+1}: \mathrm{F}^{\mathrm{p}, \mathrm{q}} \mapsto \Omega \wedge \mathrm{F}^{\mathrm{p}, \mathrm{q}}$, where, explicitly

$$
\left.\Omega \wedge F^{p, q}=\sum_{j=1}^{n} e_{j} \wedge e_{n+j} \wedge F^{p, q}=(-2 i) \sum_{j=1}^{n} \mathfrak{f}_{j}^{\dagger} \wedge \mathfrak{f}_{j} \wedge F^{p, q}=2 i \partial_{\underline{z}} \wedge\right\rceil F^{p, q}
$$

The L-operator enjoys the properties listed in the proposition below (see also [21]).

Proposition 5.3. One has

(i) $\left[\mathrm{L}, \partial_{\underline{X}} \wedge\right]=0,\left[\mathrm{~L}, \partial_{\underline{X}} \bullet\right]=-\partial_{\underline{X} \mid} \wedge$

and also

(ii) $\left[\mathrm{L}, \partial_{\underline{z}^{\dagger}} \wedge\right]=0,\left[\mathrm{~L}, \partial_{\underline{z}} \wedge\right]=0$

(iii) $\left[\mathrm{L}, \partial_{\underline{z}^{\dagger}} \bullet\right]=i \partial_{\underline{z}^{\dagger}} \wedge,\left[\mathrm{L}, \partial_{\underline{z}} \bullet\right]=-i \partial_{\underline{z}} \wedge$

The counterpart of the L-operator is the $\Lambda$-operator.

Definition 5.4. The $\Lambda$-operator is defined as $\Lambda: \mathcal{E}^{p, q} \longrightarrow \mathcal{E}^{p-1, q-1}: F^{p, q} \mapsto \Lambda F^{p, q}$, where, explicitly

$$
\Lambda \mathrm{F}^{\mathrm{p}, \mathrm{q}}=\sum_{j=1}^{\mathrm{n}} \mathrm{e}_{\mathrm{j}} \bullet \mathrm{e}_{\mathrm{n}+\mathrm{j}} \bullet \mathrm{F}^{\mathrm{p}, \mathrm{q}}=(-2 \mathrm{i}) \sum_{j=1}^{\mathrm{n}} \mathrm{f}_{j}^{\dagger} \bullet \mathrm{f}_{j} \bullet \mathrm{F}^{\mathrm{p}, \mathrm{q}}=2 i \partial_{\underline{z}} \bullet \mid F^{p, q}
$$

It shows the following properties (see also [21]).

Proposition 5.5. One has

(i) $\left[\Lambda, \partial_{\underline{X}} \wedge\right]=-\partial_{\underline{X}} \bullet,\left[\Lambda, \partial_{\underline{X}} \bullet\right]=0$

and also

(ii) $\left[\Lambda, \partial_{\underline{z}^{\dagger}} \wedge\right]=i \partial_{\underline{z}^{\dagger}} \bullet,\left[\Lambda, \partial_{\underline{z}} \wedge\right]=-i \partial_{\underline{z}} \bullet$

(iii) $\left[\Lambda, \partial_{\underline{z}^{\dagger}} \bullet\right]=0,\left[\Lambda, \partial_{\underline{z}} \bullet\right]=0$

A rather tedious computation leads to the commutator of the $L$ and $\Lambda$ operators. Proposition 5.6. One has

$$
[\mathrm{L}, \Lambda] \mathrm{F}^{\mathrm{p}, \mathrm{q}}=(\mathrm{n}-\mathrm{p}-\mathrm{q}) \mathrm{F}^{\mathrm{p}, \mathrm{q}}
$$

Finally, putting for an arbitrary multivector function $F \equiv \sum_{p=0}^{n} \sum_{q=0}^{n} F^{p, q}$ :

$$
\left.\left.H[F]=\sum_{p=0}^{n} \sum_{q=0}^{n}(n-p-q) F^{p, q}=\left(n-2\left(\partial_{\underline{z}^{\dagger}} \wedge\right\rfloor-\partial_{\underline{z}} \wedge\right\rfloor\right)\right) F
$$

we obtain the following relations (see also [20]). 
Proposition 5.7. One has

(i) $[\mathrm{L}, \Lambda]=\mathrm{H}$

(ii) $[\mathrm{H}, \Lambda]=2 \Lambda$

(iii) $[\mathrm{H}, \mathrm{L}]=-2 \mathrm{~L}$

meaning that the operators $(L, \Lambda, H)$ generate the Lie algebra $\mathfrak{s l}_{\mathbb{C}}(2)$

\section{The Hodge "star"-operator}

The Hodge $*$-operator for smooth real differential forms in $\mathbb{R}^{m}$ may be defined as follows (see e.g. [19, 24]).

Definition 6.1. Let $\left\{\mathfrak{j}_{1}, \ldots, \mathfrak{j}_{\mathrm{r}}\right\} \cup\left\{\mathfrak{j}_{\mathrm{r}+1}, \ldots, \mathfrak{j}_{\mathbf{m}}\right\}=\{1, \ldots, \mathrm{m}\}$ and $\left\{\mathfrak{j}_{1}, \ldots, \mathfrak{j}_{\mathrm{r}}\right\} \cap\left\{\mathfrak{j}_{\mathrm{r}+1}, \ldots, \mathfrak{j}_{\mathbf{m}}\right\}=\emptyset$, with $j_{1}<\ldots<j_{r}$. Then

$$
*\left(d X_{j_{1}} \wedge \cdots \wedge d X_{j_{r}}\right)=\sigma d X_{j_{r+1}} \wedge \cdots d X_{j_{m}}
$$

where $\sigma$ is the signature of the permutation $\left(\mathfrak{j}_{r+1}, \ldots, \mathfrak{j}_{m}, \mathfrak{j}_{1}, \ldots, \mathfrak{j}_{r}\right)$.

It constitutes an isomorphism $*: \bigwedge^{r} \longrightarrow \bigwedge^{m-r}$, its inverse being given by

$$
*^{-1}=(-1)^{r(m-r)} *
$$

which implies that

$$
*^{2}=(-1)^{r(m-r)}
$$

By means of this $*$-operator the Hodge co-derivative $\mathrm{d}^{*}$ may be expressed in terms of the derivative $\mathrm{d}$ as

$$
d^{*} \omega^{r}=(-1)^{r} * d *^{-1} \omega^{r}=(-1)^{r(m+1-r)} * d * \omega^{r}
$$

In the actual case of even dimension $(m=2 n)$ we find that the Hodge star-operator is an isomorphism $*: \bigwedge^{\mathfrak{p}, \mathrm{q}} \longrightarrow \bigwedge^{\mathfrak{n}-\mathrm{q}, \mathrm{n}-\mathrm{p}}$ for which $*^{-1}=(-1)^{(\mathfrak{p}+\mathrm{q})^{2}} *$ and thus $*^{2}=(-1)^{(\mathfrak{p}+\mathrm{q})^{2}}$. For the Hodge derivative and co-derivative we then obtain

$$
\mathrm{d}^{*}=* \mathrm{~d} * \quad \text { and } \quad \mathrm{d}=* \mathrm{~d}^{*} *
$$

and similarly for the twisted versions

$$
\left.\mathrm{d}\right|^{*}=* \mathrm{~d} \mid * \quad \text { and } \quad \mathrm{d}\left|=* \mathrm{~d}^{*}\right| *
$$

Simply applying the conversion rules of the foregoing section we obtain the counterparts of these relations in the setting of multivector functions, involving then the Dirac operators.

Proposition 6.2. One has 
(i) $\partial \underline{x} \bullet=*\left(\partial_{\underline{x}} \wedge\right) *$ and $\partial \underline{x} \wedge=*(\partial \underline{x} \bullet) *$

(ii) $\partial_{\underline{x}} \bullet=*\left(\partial_{\underline{X}} \wedge\right) *$ and $\partial_{\underline{X}} \wedge=*(\partial \underline{\underline{x}} \bullet \bullet *$

and also

(iii) $\partial_{\underline{z}} \bullet=*\left(\partial_{\underline{z}} \wedge\right) *$ and $\partial_{\underline{z}} \wedge=*\left(\partial_{\underline{z}} \bullet\right) *$

(iv) $\partial_{\underline{z}^{\dagger}} \bullet=*\left(\partial_{\underline{z}^{\dagger}} \wedge\right) *$ and $\partial_{\underline{z}^{\dagger}} \wedge=*\left(\partial_{\underline{z}^{\dagger}} \bullet\right) *$

Clearly, we may convert Proposition 6.2 back to the differential form setting.

Proposition 6.3. One has

(i) $\partial^{* \mathrm{c}} \bullet=*(\partial \wedge) *$ and $\partial \wedge=*\left(\partial^{* c} \bullet\right) *$

(ii) $\partial^{*} \bullet=*\left(\partial^{\mathrm{c}} \wedge\right) *$ and $\partial^{\mathrm{c}} \wedge=*\left(\partial^{*} \bullet\right) *$

Of course, it is also possible to express the Hodge star operator in $\mathbb{R}^{m}$ directly in the Clifford algebra setting; Definition 6.1 is then converted as follows (see [6]):

$$
*\left(e_{j_{1}} \cdots e_{j_{r}}\right)=(-1)^{\frac{r(r+1)}{2}} e_{M} e_{j_{1}} \cdots e_{j_{r}}
$$

where $e_{M}$ is the so-called pseudoscalar given by $e_{M}=e_{1} \cdots e_{m}$, of which the square equals $e_{M}^{2}=(-1)^{\frac{m(m+1)}{2}}$. It follows that for 1 -vectors the $*$-operation reduces to a multiplication from the left by $-e_{M}$. Also $* 1=e_{M}$ and $* e_{M}=1$.

In the Hermitean case with even dimension $m=2 n$, let us compute $e_{M}$ in terms of the Witt basis vectors. We consecutively obtain

$$
\begin{aligned}
e_{M} & =\prod_{\alpha=1}^{2 n} e_{\alpha}=\prod_{j=1}^{n}\left(\mathfrak{f}_{j}-\mathfrak{f}_{j}^{\dagger}\right) \prod_{j=1}^{n} i\left(\mathfrak{f}_{j}+\mathfrak{f}_{j}^{\dagger}\right) \\
& =i^{n}(-1)^{\frac{n(n-1)}{2}} \prod_{j=1}^{n}\left(\mathfrak{f}_{j}-\mathfrak{f}_{j}^{\dagger}\right)\left(\mathfrak{f}_{j}+\mathfrak{f}_{j}^{\dagger}\right)=i^{n}(-1)^{\frac{n(n-1)}{2}} \prod_{j=1}^{n}\left(\mathfrak{f}_{j} \mathfrak{f}_{j}^{\dagger}-\mathfrak{f}_{j}^{\dagger} \mathfrak{f}_{j}\right) \\
& =2^{n} i^{n}(-1)^{\frac{n(n-1)}{2}} \mathfrak{f}_{1} \wedge \mathfrak{f}_{1}^{\dagger} \wedge \mathfrak{f}_{2} \wedge \mathfrak{f}_{2}^{\dagger} \wedge \cdots \wedge \mathfrak{f}_{\mathfrak{n}} \wedge \mathfrak{f}_{n}^{\dagger}
\end{aligned}
$$

showing that the pseudoscalar has bidegree $(n, n)$.

As an example we have, for $m=4, n=2$, that the images under the $*$-operation of the Euclidean basis vectors are 3 -vectors given by

$$
* e_{1}=-e_{2} e_{3} e_{4}, \quad * e_{2}=e_{1} e_{3} e_{4}, \quad * e_{3}=-e_{1} e_{2} e_{4}, \quad * e_{4}=e_{1} e_{2} e_{3}
$$


The Witt basis vectors $\mathfrak{f}_{1}$ and $\mathfrak{f}_{2}$, of bidegree $(0,1)$, transform into $(1,2)$-multivectors:

$$
\begin{aligned}
& * \mathfrak{f}_{1}=-2 \mathfrak{f}_{1} \wedge \mathfrak{f}_{2} \wedge \mathfrak{f}_{2}^{\dagger} \\
& * \mathfrak{f}_{2}=-2 \mathfrak{f}_{1} \wedge \mathfrak{f}_{1}^{\dagger} \wedge \mathfrak{f}_{2}
\end{aligned}
$$

while $\mathfrak{f}_{1}^{\dagger}$ and $\mathfrak{f}_{2}^{\dagger}$, of bidegree $(1,0)$, transform into $(2,1)$-multivectors:

$$
\begin{aligned}
& * \mathfrak{f}_{1}^{\dagger}=2 \mathfrak{f}_{1}^{\dagger} \wedge \mathfrak{f}_{2} \wedge \mathfrak{f}_{2}^{\dagger} \\
& * \mathfrak{f}_{2}^{\dagger}=2 \mathfrak{f}_{1} \wedge \mathfrak{f}_{2} \wedge \mathfrak{f}_{2}^{\dagger}
\end{aligned}
$$

\section{$7 \quad$ Afterword}

In the previous sections we established and illustrated a "natural" isomorphism between on the one hand the algebra of complex differential forms (extended with the Hodge star operator and the inner product or dot product) with the underlying structure of a Grassmann algebra, and on the other hand the algebra of multi-vector functions in Hermitean Clifford analysis with the underlying structure of a complex Clifford algebra. The Hermitean Dirac operators, underlying the notion of Hermitean monogenicity, may well be identified with the Kählerian derivatives for complex differential forms, one of which is the famous $\bar{\partial}$ operator from several complex variables theory. It should be emphasized, as was done from the beginning, that only differential forms in $\mathbb{C}^{\mathrm{n}}$ or in open regions thereof were considered, and that actually Hermitean Clifford analysis was developed only in flat space $\mathbb{C}^{n}$. As was also mentioned Hermitean Clifford analysis on curved Kählerian manifolds is the subject of the forthcoming paper [11. Finally this paper is by no means a plea for substituting Hermitean multivector functions for complex differential forms. Both worlds, how convincing the similarities might be, have their own interest en properties; this paper intended to illustrate the very close connections between Hermitean Clifford analysis and complex analysis and the benefits obtained from exchanging knowledge between both.

Received: December 2009. Revised: April 2010.

\section{References}

[1] F. Brackx , J. Bureš, H. De Schepper, D. Eelbode, F. Sommen and V. Souček, Fundaments of Hermitean Clifford Analysis. Part I: Complex structure, Compl. Anal. Oper. Theory 1(3), 2007, 341-365.

[2] F. Brackx, J. Bureš, H. De Schepper, D. Eelbode, F. Sommen and V. Souček, Fundaments of Hermitean Clifford Analysis. Part II: Splitting of h-monogenic equations, Complex Var. Elliptic Eq. 52(10-11), 2007, 1063-1079.

[3] F. Brackx, B. De Knock and H. De Schepper, A matrix Hilbert transform in Hermitean Clifford analysis, J. Math. Anal. Appl. 344 (2008), 1068-1078. 
[4] F. Brackx, B. De Knock, H. De Schepper and F. Sommen, On Cauchy and MartinelliBochner integral formulae in Hermitean Clifford analysis, Bull. Braz. Math. Soc. New Series 40(3), 2009, 395-416.

[5] F. Brackx, R. Delanghe and F. Sommen, Clifford Analysis, Pitman Publishers, BostonLondon-Melbourne, 1982.

[6] F. Brackx, R. Delanghe and F. Sommen, Differential forms and/or multivector functions, Cubo 7(2), 2005, 139-169.

[7] F. Brackx, H. De Schepper, D. Eelbode and V. Souček, The Howe dual pair in Hermitean Clifford analysis, accepted for publication in Rev. Mat. Iberoam. 26(2), 2010, 449479 .

[8] F. Brackx, H. De Schepper and F. Sommen, A theoretical framework for wavelet analysis in a Hermitean Clifford setting, Comm. Pure Appl. Anal. 6(3), 2007, 549-567.

[9] F. Brackx, H. De Schepper and F. Sommen, The Hermitian Clifford analysis toolbox, Appl. Clifford Algebras 18(3-4), 2008, 451-487.

[10] F. Brackx, H. De Schepper and V. Souček, On the Structure of Complex Clifford Algebra Adv. Appl. Clifford Alg. DOI: 10.1007/s0006-010-0270-4.

[11] F. Brackx, H. De Schepper and V. Souček, Hermitean Clifford Analysis on Kählerian manifolds (in preparation).

[12] A. Damiano, D. Eelbode and I. Sabadini, Invariant syzygies for the Hermitian Dirac operator, Math. Zeitschrift 262, 2009, 929-945.

[13] R. Delanghe, R. LÁviČKa and V. SouČEk, On polynomial solutions of generalized MoisilThéodoresco systems and Hodge-de Rham systems (arXiv: 0908.0842).

[14] R. Delanghe, R. LÁviČKa and V. SoučEk, The Fischer decomposition for Hodge-de Rham systems in Euclidean spaces (arXiv: 1012.4994).

[15] R. Delanghe, F. Sommen and V. Souček, Clifford Algebra and Spinor-Valued Functions, Kluwer Academic Publishers, Dordrecht, 1992.

[16] D. Eelbode, Stirling numbers and spin-Euler polynomials, Exp. Math. 16(1) (2007), 55-66.

[17] J. Gilbert and M. Murray, Clifford Algebras and Dirac Operators in Harmonic Analysis, Cambridge University Press, Cambridge, 1991.

[18] K. Gürlebeck, K. Habetha and W. Sprössig, Holomorphic Functions in the Plane and n-dimensional Space, Birkhäuser Verlag, Basel, 2008.

[19] K. Maurin, Analysis, part II, D. Reidel Publishing Company, Dordrecht - Boston - London, PWN-Polish Scientific Publishers, Warszawa, 1980. 
[20] M. L. Michelsohn, Clifford and Spinor Cohomology of Kähler Manifolds, American Journal of Mathematics 102(6) (1980), 1083-1146.

[21] A. Moroianu, Lectures on Kähler geometry, London Mathematical Society Student Texts 69, Cambridge University Press (Cambridge, 2007).

[22] I. R. Porteous, Topological Geometry, Van Nostrand Reinhold Company, London - New York - Toronto - Melbourne, 1969.

[23] I. Sabadini and F. Sommen, Hermitian Clifford analysis and resolutions, Math. Meth. Appl. Sci. 25 (16-18) (2002), 1395-1414.

[24] C. von Westenholz, Differential Forms in Mathematical Physics, Stud. Math. Appl., vol 3, North-Holland, Amsterdam, 1978. 